\title{
Histone code dictates fate biasing of neural crest cells to melanocyte lineage
}

\author{
Desingu Ayyappa Raja ${ }^{1,2}$, Yogaspoorthi Subramaniam ${ }^{1,2}$, Vishvabandhu Gotherwal ${ }^{1,2}$, \\ Jyoti Tanwar ${ }^{1,2}$, Rajender Motiani ${ }^{1}$, Sridhar Sivasubbu ${ }^{1}$, Rajesh S Gokhale ${ }^{1,3 \$}$ and \\ Vivek T Natarajan ${ }^{1,2, *}$
}

\footnotetext{
${ }^{1}$ CSIR-Institute of Genomics of Genomics and Integrative Biology, Mathura Road, New Delhi, India.

${ }^{2}$ Academy of Scientific and Innovative Research, New Delhi, India

${ }^{3}$ National Institute of Immunology, Aruna Asaf Ali Marg, New Delhi, India.

\$ present address

*Correspondence must be addressed to:

Vivek T Natarajan, PhD.,

CSIR-Institute of Genomics and Integrative Biology

Mathura Road, New Delhi-110020

Email:tnvivek@igib.in,tn.vivek@igib.res.in
}

Key Words: histone variant, melanocyte, gene regulatory network, epigenetic regulation, fate-bias, specification, pigmentation, histone code

Running Title: H2A.Z.2 dictates melanocyte fate

\section{Summary statement}

Here we report mechanism behind the critical role of Histone variant H2A.Z.2 in fate allocation and subsequent commitment of melanocyte identity, from a multipotent state. 


\begin{abstract}
In the neural crest lineage, progressive fate-restriction and stem cell assignment are critical for both development and regeneration. While the fate-commitment events have distinct transcriptional footprints, fate-biasing is often transitory and metastable, and is thought to be moulded by epigenetic programs. Hence molecular basis of specification is difficult to define. In this study, we establish a role of a histone variant H2a.z. 2 in specification of melanocyte lineage from multipotent neural crest cells. Silencing of H2a.z. 2 reduces the number of melanocyte precursors in developing zebrafish embryos, and from mouse embryonic stem cells in vitro. We demonstrate that this histone variant occupies nucleosomes in the promoter of key melanocyte determinant Mitf, and enhances its induction. CRISPRCas9 based targeted mutagenesis of this gene in zebrafish drastically reduces adult melanocytes, as well as their regeneration. Thereby our study establishes a histone based specification code upstream to the core gene regulatory network in the neural crest lineage of melanocytes. This epigenetic code renders a poised state to the promoter of key determinant and enhances activation by external instructive signals thereby establishing melanocyte fate identity.
\end{abstract}




\section{Introduction}

Cell fate determination is driven by a cascade of transcription factors that are in-turn governed by external cues (Kawakami and Fisher, 2011; Martik and Bronner, 2017). The multipotent neural crest sets a paradigm for stem cell pluripotency and lineage specification, and serves as a model to understand fate decisions. Recent data emerging from single-cell transcriptomics of neural crest cells (NCCs) demonstrates emergence of fate bias or specification that further unfolds to culminate in fate commitment (Soldatov et al., 2019) or determination. NCC's and NCC-derived progenitor population give rise to melanocytes during embryonic development, and adult skin homeostasis and regeneration respectively (White and Zon, 2008). Ability to trace cells visually, combined with the extensive literature on gene regulatory network, render melanocytes as a preferred choice to trace mechanisms behind fate decisions.

Almost all aspects of melanocyte biology, from lineage commitment to differentiation, is controlled by the central transcription factor MITF (Goding and Meyskens, 2006; Kawakami and Fisher, 2017). WNT and BMP signalling (Jin et al., 2001; Takeda et al., 2000), and downstream transcription factors SOX10 (Marathe et al., 2017), PAX3 (Watanabe et al., 1998), FOXD3 (Kos et al., 2001) and LEF1 (Dunn et al., 2000), form the core of gene regulatory network that control Mitf induction and mediate melanocyte specification and determination. While the upstream instructive signals and downstream orchestrators are well appreciated, cell intrinsic modulators that mediate specification events remain obscure. Epigenetic mechanisms, by altering chromatin accessibility, could provide an intervening regulatory layer and are likely to play a key role in determining the fate of NCC lineages.

Epigenetic regulators including histone deacetylase HDAC1 (Ignatius et al., 2008; Ignatius et al., 2013) involved in locus specific histone deacetylation, DNA methyl transferase DNMT3b, that causes base methylation (Rai et al., 2010), and histone variant H3.3 that replaces the core histone H3, are involved in the derivation of specific cell types from NCCs (Cox et al., 2012). Another histone variant H2A.Z, encoded by two genes H2a.z. 1 and H2a.z. 2, has been implicated in NCC differentiation (Punzeler et al., 2017; Sivasubbu et al., 2006). Recent studies demonstrate H2A.Z to play a crucial role in human metastatic melanoma survival and proliferation (Vardabasso et al., 2015) and involvement of H2A.Z chaperone PWWP2A in NCC differentiation (Punzeler et al., 2017). Since melanoma recapitulates neural crest fate, (Kaufman et al., 2016), it is possible that histone variants dictate the fate of melanocytes from neural crest derived progenitors.

In this study, using zebrafish as well as mouse embryonic stem cell-derived melanocyte models we demonstrate a selective role for H2A.Z.2 in fate-biasing of NCCs to melanocytes. Further, we show that this variant occupies the promoter of Mitf and upstream regulator Sox 9 , and governs the inducibility of Mitf under appropriate cues. A series of CRISPR targeted mutations in h2a.z. 2 reinforce its central 
role during pigmentation and the related process of melanocyte regeneration in adult zebrafish. Thereby, we establish a histone code upstream to the transcription factor network that enables cell-intrinsic responsiveness to external instructive signals. Our study adds a new dimension to the recent developments in tracing the lineage map of NCC derivatives and provides a molecular basis to the fatebias observed during specification.

\section{Results}

\section{Histone variant $h 2 a_{0} . z_{.} 2$ but not $h 2 a_{.} z_{.} 1$ is involved in the derivation of melanocytes in zebrafish}

Across vertebrates, the histone variant H2A.Z is encoded by two distinct paralogs H2A.Z.1 (Z1) and H2A.Z.2 (Z2), that are 97\% identical at the protein level, and hence difficult to distinguish experimentally (Dryhurst et al., 2009; Matsuda et al., 2010). Disruption of Z1 is not compensated by intact Z2 and the knockout mouse embryos fail to survive (Faast et al., 2001). Therefore, to decipher their individual contribution to the differentiation of neural crest cells (NCCs), especially to melanocytes, we adopted a morpholino based transient knockdown strategy in developing zebrafish embryos. Upon knock down of Z1, gross embryonic deformities and poor development were observed, as has also been reported previously (Madakashira et al., 2017; Murphy et al., 2018; Sivasubbu et al., 2006). Z2 morphants only had milder effects on gross morphology, and they presented a drastic reduction in pigmentation (Fig 1A-D).

Pigmentation phenotype of Z2 MO could be recapitulated using splice block MO targeted to $\mathrm{Z} 2$, and also upon co-injection of Z2 MO with p53 MO, reiterating the specific nature of the phenotype (Supplementary Fig 1). Upon co-injection of Z2 MO along with mouse H2a.z.1 or H2a.z.2 mRNAs, a rescue of around 50\% was observed only for Z2 mRNA (Fig 1E). Thereby demonstrating that Z2 has a distinct pigmentation role, and is not compensated by increased dosage of Z1.

\section{h2a.z. 2 depletion selectively reduces melanocyte numbers}

The characteristic reduction in pigmentation of Z2 morphants could be either due to decreased pigment production or due to the absence of mature pigmented cells. At 2 days post fertilization ( $2 \mathrm{dpf})$, we observed a severe reduction in the number of ftyrp1:GFP + ve cells in Z2 morphants, notably in dorsal as well as the ventral pool of melanocytes above the yolk sac, clearly indicating that the number of melanocytes is affected (Fig 1B). Additionally at $6 \mathrm{dpf}$, the lateral line melanocytes were reduced in Z2 morphants, suggesting that the progenitor pool may be affected (Fig 1D). We therefore used various transgenic reporter lines from neural crest to melanocyte lineage to estimate respective cell numbers at different stages of development as indicated in (Fig 1F). We observed that Z2 morphants displayed approximately $45-50 \%$ reduction in the differentiating/differentiated melanophores and a $30 \%$ reduction in melanoblast numbers (Fig 1F). However, the sox10 +ve progenitor population remained 
unaffected in Z2 morphants. h2a.z.2 is therefore likely to play a key role in the lineage commitment of melanocytes from neural crest derived progenitors.

\section{h2a.z.2 dictates melanocyte and glial footprint in NCCs}

Multipotent NCCs progressively undergo the process of fate specification and determination to give rise to melanocytes, craniofacial cartilage, adipocytes, smooth muscle cells, schwann cells and neurons (Donoghue et al., 2008; Le Douarin et al., 2004). Based on Whole mount In Situ Hybridization (WISH), we observed that the early neural crest gene expression of foxd3, sox 10 and tfap $2 a$ were not decreased in Z2 morphants (Fig 1G\&H). This indicates that depletion of h2a.z.2 could selectively affect a subpopulation of NCC derived progenitors from which melanocytes and possibly a few other cell types would be derived. Hence we resorted to identify gene expression changes in this seemingly homogeneous population for alterations that could explain decreased melanocyte numbers.

We created Z2 morphants in $T$ (sox 10:GFP) line and sorted the GFP +ve cells at 16-18 hpf. At this time point, neural crest derived sox 10 expression is retained and also lineage specific differentiation markers begin to emerge and the cell fate identity is established (Curran et al., 2010; Wagner et al., 2018). Microarray and pathway enrichment analysis of the GFP + ve cells revealed that the set of downregulated genes mapped to developmental pigmentation related processes (Fig 2A-C). Targeted analysis of the data indicated reduced expression of transcription factors (TFs) with known role in melanocyte lineage, such as mitfa, tfap2a, tfap2e and sox $9 b$, as well as melanocyte migration and survival factors such as kita and ednrbla. Similarly, we also observed decreased expression of glial lineage transcription factors such as $n f a t c 4, \operatorname{pax} 7, \operatorname{lmo} 4$ etc. However, the neuronal lineage TFs such as foxol, foxo 3 and sox 8 were upregulated in the Z2 morphants (Fig 2D). As these are gene expression changes in the isolated sox $10+v e$ cells, we interpret the decreased expression to indicate alterations in the subpopulations of differentiating cells derived from the NCCs.

To verify the reduction in specific cell types, we adopted WISH for lineage specific differentiation markers and analysis of cell type specific transgenic lines that label NCC derivatives. $d c t$ and kita that label melanocytes, show decreased staining in the Z2 morphants (Fig 2E). Similar pattern of decreased staining was observed for differentiated schwann cells using markers krox20 and $m b p$ that labelled the lateral line associated glial cells (Fig 2F). Undifferentiated schwann cells, visualized by $\operatorname{Tg}\left(f_{0 x D}\right.$ 3:GFP), showed substantial reduction. $\operatorname{Tg}(n b t: D s R e d)$ marked enetric neurons remain unaffected (Supplementary Fig 2) and similarly WISH for isl2a showed no change (Fig $2 \mathrm{G}$ bottom panel). However, we observed the presence of ectopic neurons in the trunk, above the spinal chord neuron bundle, suggestive of a local increase in the neuronal population (Fig 2G). Labelling by $\operatorname{Tg}(\operatorname{sox} 10: G F P)$ as well as alcian blue staining of craniofacial appendages and fin cartilage showed no major changes, suggesting normal cartilage specification. However, we noticed a consistent decrease in jaw size as a result of defective ceratobranchial appendage spacing (Supplementary Fig 2). Together, 
our data suggests that h2a.z.2 plays a selective role in the derivation of melanocytes and glial populations from the NCC. Interestingly, previous studies have shown that these two cell types emerge from a common progenitor (Raible and Eisen, 1994).

\section{$h 2 a . z .2$ functions in conjunction with mitf to control melanocyte derivation}

Micropthalmia associated transcription factor (Mitf), though seemingly dispensible for specification (Johnson et al., 2011), is the earliest known marker of lineage commitment and controls expression of downstream identity genes to enable melanocyte fate determination (Goding, 2000; Lister et al., 1999). We indeed observe a decrease in mitfa expression in sox10+ve cells, and this was further corroborated by WISH staining for mitfa (Fig 2H). Therefore we set out to decipher the interplay of h2a.z.2 and mitfa in mediating melanocyte lineage commitment. A marked rescue in the melanocyte counts in mitfa mRNA injected Z2 morphants was noted (Fig I\&J). The above data, confirms genetic interaction between $h 2 a . z .2$ and mitfa, and h2a.z.2 likely functions upstream of mitfa and hence could modulate melanocyte fate.

\section{Derivation of melanocyte from mouse embryonic stem cells is under the control of $H 2 a . z .2$}

From these experiments it is likely that H2a.z.2 plays a role in the specification/determination of an upstream progenitor population from which melanocytes arise. Additionally, decrease in glia in $h 2 a . z .2$ morphant indicates that the bipotent progenitor of melanocytes and glial populations could be affected. Further, an increase in neurons is suggestive of a compensatory increase in neuronal progenitors. Our observations therefore indicate a heirarchical model of fate determination in the NCCs, which is in tune with recent studies (Le Douarin and Dupin, 2003; Raible and Eisen, 1994; Soldatov et al., 2019). We propose a model, wherein $h 2 a . z .2$ would operate at the level of the tripotent glial/neuronal/melanocyte progenitor in specifying a melanocyte/glial fate (Fig $2 \mathrm{~K})$.

While the zebrafish based experiments helped us to narrow down the function of this variant in melanocyte derivation, cellular and molecular insights are required to establish its role in specification. To understand this molecular mechanistic link, we established a method to derive melanocytes from $\mathrm{R} 1 / \mathrm{E}$ mouse embryonic stem cells (mESCs), wherein the mESCs were converted to melanocytes through an intermediate embryoid body state (EBs) (Fig 3A). Targeted gene expression analysis of NCC, melanocyte, and stemness related genes were performed at different days of melanocyte formation (Fig 3B). As anticipated, the expression of Mitf was induced at day 5 and further increased till day 16. Similarly, we observed the expression of pigmentation gene $D c t$ to be induced during the course of experiment. We noticed that the expression of stemness factors Oct4 and Nanog were drastically reduced during the course of induction, suggesting that the cells are undergoing differentiation. Strikingly, we noticed that the expression patterns of H2a.z. 2 and H2a.z. 1 were opposite to each other. While the expression of H2a.z.2 was positively correlated with melanocyte specification, 
the expression of H2a.z. 1 was decreased. This pattern of H2a.z.1 is similar to stemness factors Oct4 and Nanog.

To illustrate the functional role of H2a.z.2 in mESCs, we silenced H2a.z.2 and studied melanocyte derivation. In the cells stably expressing short hairpin RNA that targets h2a.z.2 (shZ2), a reduction of around 50\% in H2a.z. 2 mRNA levels could be observed, while H2a.z.1 levels remained unaltered (Fig 3C). Interestingly, previous studies report that knockdown of H2a.z. 1 in embryonic stem cells leads to the formation of defective embryoid bodies with irregular structural integrity (Hu et al., 2013). We did not observe significant difference in the size and morphology of the H2a.z.2 depleted EBs (Fig 3D), further strengthening a differential role for the two proteins, at least during early developmental stages.

Upon induction of melanocytes from R1/E cells stably expressing shZ2, compared to nontargetting shRNA (shNT), the expression of neural crest gene Sox10 remained unaltered. This observation is strikingly similar to Z2 MO scenario in zebrafish embryos (Fig 3E). A significant decrease in the mRNA level of the master transcription factor Mitf and its target gene Tyr in shZ2 cells as compared to shNT cells was observed. Thus highlighting a conserved role for H2a.z.2 in melanocyte specification across zebrafish and mouse. Having set up the cell based model of melanocyte specification and the effect of H2a.z.2 in derivation of melanocytes in this model, we set out to identify effectors and the mechanism by which H2a.z.2 could mediate melanocyte specification.

\section{H2A.Z.2 occupies Sox9 and Mitf promoters, and controls Mitf inducibility}

Both H2A.Z.1 and H2A.Z.2 occupy the nucleosomes in promoter as well as enhancer regions immediate to the transcription start site (TSS) of the downstream genes. This occupancy can either facilitate (Bargaje et al., 2012; Gevry et al., 2009; Zovkic et al., 2014) or repress (Dai et al., 2017; Hardy et al., 2009) transcription, in a context and a model system dependent manner. Partly, this discrepancy could be attributed to the near identical proteins that are indistinguishable in chromatin immunoprecipitation (ChIP) studies and to the complex nature of chromatin changes brought about by H2A.Z. We therefore employed a silencing strategy followed by ChIP to address the molecular mechanism selective to Z2.

H2A.Z ChIP followed by q-PCR for the promoter proximal nucleosomes of Mitf, and other critical upstream effectors Sox 9 , Sox 10 and Ckit was performed across various stages of melanocyte specification in the mESC model. We then compared the nucleosome occupancy of H2A.Z to their mRNA level changes at these stages of differentiation. All these four promoters showed increased occupancy during the course of melanocyte formation from mESCs. Interestingly, we observed that mRNA expression of Mitf, positively correlated with the occupancy of H2A.Z (H2A.Z.1+H2A.Z.2) in its promoter, suggesting that H2A.Z facilitates induction of Mitf expression possibly by modulating the accessibility of Mitf promoter (Fig 3H). 
Though our progressive induction model provided a window to assess kinetic changes in promoter occupancy, heterogeneity in the cell populations arising during the course of melanocyte induction, limited our interpretations. Therefore, we employed a complementary approach wherein we utilized B16 melanoma cells that would represent specified melanocytes. ChIP with H2A.Z antibody was carried out in B16 cells to check the occupancy in promoter regions of genes related to Mitf, and upstream regulators as well as genes downstream to it in melanocytes. Mitf and Sox 9 promoters showed considerable enrichment of 5-7\% over input (Fig 3I), suggesting that these genes might be under H2A.Z control. In contrast, several pigmentation genes like Dct, Tyr, Tyrp 1, Sox10 etc. were not enriched for H2A.Z in B16 cells. This is in tune with previous studies on H2A.Z in human melanoma cells (Vardabasso et al., 2015) and our meta-analysis further corroborated this observation (Supplementary Fig 3).

Knockdown of H2a.z.2 in B16 cells with siRNA resulted in a consistent $70-80 \%$ decrease in mRNA levels of H2a.z.2, while H2a.z.1 levels remained unchanged (Supplementary Fig 3). H2A.Z ChIP-qPCR was carried out for Mitf, Sox9 and Sox10 promoter regions in H2a.Z.2 knockdown B16 cells (Fig 3J,L\&N). We observed decreased occupancy of H2A.Z upon Z2 knockdown (Fig 3L\&N) and a correlated decrease in mRNA expression of Mitf and Sox9 (Fig 3M\&O) suggested a direct consequence of Z2 incorporation. In contrast, Sox10 promoter, which had minimal occupancy in B16 cells, showed no change upon H2a.z.2 silencing. Correspondingly, the Sox10 mRNA levels remained unaltered (Fig 3K). Promoter specific luciferase constructs, further demonstrated Mitf and Sox 9 promoters to be regulated by H2A.Z.2 (Supplementary Fig 3). Taking into account these observations we conclude that $\mathrm{Z} 2$ modulates the promoter region of MITF and facilitates its induction, the absence of which leads to decreased Mitf expression resulting in reduced number of specified melanocytes.

The effect of H2A.Z2 occupancy on Mitf promoter was addressed in reporter assay with the overexpression of H2a.Z.2 in B16 cells. While there was no change in the Mitf promoter activity under basal conditions in Z2 overexpressing cells, MSH stimulation resulted in 7-fold upregulation, which was much higher than control empty vector transfected cells (Fig 3P\&Q). We thus conclude that positioning of H2A.Z.2 upstream of Mitf facilitates its induction by appropriate cues. Occupancy of H2A.Z.2 renders chromatin state of the promoter to be highly responsive to activating signals in the form of transcription factors. The role of H2A.Z.2 delineated in the current study shows striking similarity with its role in brain, wherein incorporation of H2A.Z mediates dynamic gene expression changes associated with memory formation (Zovkic et al., 2014). It also is interesting to note that upon Z2 silencing in the zebrafish system as well, $\operatorname{sox} 9 a$ and $\operatorname{sox} 9 b$ show reduction in the NCC populations (Fig 2D). Given the upstream role of Sox 9 as a positive regulator of Mitf, it is likely that this may play an additional role in melanocyte specification via Mitf. Thereby we have identified a histone code upstream of the transcription factor network that specifies melanocytes from the pluripotent NCCs (Fig $3 R)$. 


\section{$H 2 a . z .2$ is required for adult melanocyte regeneration}

Targeted knockout of H2a.z. 1 has been attempted in several organisms and found to be embryonically lethal. However, the Z2 variant has not been not systematically studied. CRISPR-Cas9 based targeted deletion of h2a.z. 2 in zebrafish resulted in severe lethality and gross deformities (Supplementary Fig 4). Genotyping of h2a.z.2 locus from these embryos revealed several in-dels resulting in large scale disruptions of this gene. Though h2a.z. 2 seems to be essential for early development, we could isolate three h2a.z. 2 mutant lines by targetting the coding region downstream to the core histone domain which map to the $\mathrm{C}$-terminal extension region beyond $\alpha \mathrm{C}$ domain (Fig $4 \mathrm{~A}$ ). This region is unstructured, and is involved in dynamic docking with histone $\mathrm{H} 3 / \mathrm{H} 4$ during nucleosome formation (Bonisch et al., 2012). Extensive work based on systematic mutagenesis of $H 2 A . Z$ (Wratting et al., 2012) and studies involving a primate specific, alternately spliced isoform H2A.Z.2.2 (Bonisch et al., 2012), enabled prediction of nucleosome stability of the mutants. The truncation of this region would have least effect on nucleosome assembly, and replacement by a shorter stretch is expected to have a moderate effect. Further, elongation of the C-terminal extension is known to have severe effect based on yeast H2A.Z study (Wratting et al., 2012). Hence the nucleosome stability would be H2A.Z.2 $2^{\text {Gln } 125 \text { fsX } 144}<$ H2A.Z.2.2 $=$ H2A.Z. $2^{\text {Ile101 fsX110 }}<$ H2A.Z. $2^{\Delta 100-120}<$ H2A.Z.2 (Fig 4A, right). We observe pigmentation phenotype severity to be in tune with the expected effect of mutations (Fig 4B).

Interestingly, in the mutant heterozygous lines we observed less melanocytes, confirming partial loss of function and a dominant nature of the mutations. In the adult animals, role of H2A.Z.2 in specification could be verified by studying melanocyte regeneration, a process wherein differentiated melanocytes arise from a pre-specified progenitor pool and could be experimentally studied using finclip experiments (Hultman et al., 2009). Herein the adult zebrafish fin is cut and allowed to regenerate for a week, during which the quiescent progenitors are stimulated and undergo the process of differentiation to generate pigmented cells. We noticed that all the three h2a.z. 2 mutants demonstrated comparable fin growth, however they displayed decreased melanocyte regeneration, in tune with the severity of the ventral stripe pigmentation observed in these mutants (Fig 4C). The NCC-derived fate specified progenitors establish in skin, and respond to melanocyte loss by providing requisite melanocytes. Decreased recall of melanocytes indicates that the progenitors are inadequately populated, and endorses the role of h2a.z.2 in melanocyte fate specification. Thereby using targetted genome engineering, we unequivocally demonstrate the role of H2A.Z.2 in establishing melanocyte progenitor population, evident not just at the embryonic stage but also during adult melanocyte regeneration.

\section{Discussion}

Decoding cell fate decision has emerged as an intense area of research, primarily due the immense application potential it has in futuristic regenerative interventions. Fate establishment is perceived to be a continuum, wherein progressive fate bias culminates in determination and is controlled by appropriate 
external signals. Currently, we understand deterministic events better, due to the characteristic gene expression signatures orchestrated by key transcription factors. However, the underlying molecular mechanisms behind fate-biasing specification events remains unexplored. Non-deterministic nature of fate bias induced during specification necessitates a modulatory role for the specifier and epigenetic factors are perfectly suited. Histone modifications and deposition of variants could function in conjunction with transcription factors and offer a greater plasticity to modulate the cell fate. In this study, we unequivocally establish the role of H2A.Z.2 as a rheostat for Mitf induction by facilitating chromatin accessibility to upstream activating signals and propose a model for melanocyte lineage specification, wherein H2A.Z.2 occupancy in Mitf acts as a key specifying signal.

Three of the fate-biasing transcriptional programs in neural crest lineage are involved in suppressing the melanocyte fate. These include Sox2 (Adameyko et al., 2012) and Neurog2 (Soldatov et al., 2019) from neural, and FoxD3 (Johnson et al., 2011) from the glial lineage, all of which actively suppress Mitf expression. H2a.z.2 in contrast is a positive enabler of melanocyte fate bias, as its overexpression facilitates Mitf induction. Heirarchical fate biasing model necessitates such opposing programs to ensure defined populations of emerging, differentiated cells. The observed concomitant decrease in the glial population suggests that H2A.Z.2 could operate as a specifier at the tripotent melanocyte/glial/neural progenitor level, driving the allocation towards a melanocyte-glial bipotent fate. Sox9 regulation by H2a.z.2 could have a dual role of promoting Mitf expression in melanocytes and also facilitating the glial specific programs (Cheung and Briscoe, 2003).

Regenerative melanocytes arise from NCC-derived stem cells that are positioned along the lateral line in close juxtaposition with dorsal root ganglia in zebrafish embryos (Dooley et al., 2013). In the adult zebrafish, precursor cells directly differentiate into mature melanocytes and also divide to yield additional lineage-restricted cells (Iyengar et al., 2015). In mammalian skin, this population resembles nerve associated melanocyte precursors (Adameyko et al., 2009) and the hair follicle bulge region progenitors, that give rise to melanocytes at every hair cycle (Nishimura et al., 2002). These progenitors are thought to restore epidermal pigmentation after wound healing and during melanocyte regeneration in conditions such as vitiligo (Chou et al., 2013).

Interestingly, though the establishment of melanocyte stem cells is seemingly independent of Mitf (Johnson et al., 2011), all the downstream events are orchestrated by this master regulator. Given this central role of Mitf in fate determination, the "melanocyte specification factor" is likely to function upstream of it. The transient epigenetic memory conferred by the incorporation of H2A.Z.2 at Mitf promoter would not just facilitate its expression upon external cues such as WNT ligands, it would also enable retention of this memory in transiently amplifying cells. The Mitf-inducing Wnt signaling pathway triggers melanocyte regeneration possibly by activating this poised state of Mitf promoter. Thereby such a histone code elucidated in our study enables retention of plasticity, a hallmark of the 
specified state. Identification of such upstream codes would enable futuristic regenerative therapeutic approaches from induced pluripotent cells, by employing selective activation of lineage determinants. 


\section{Materials and Methods}

\section{Ethics Statement}

Fish experiments were performed in strict accordance with the institutional animal ethics approval (IAEC) of the CSIR-Institute of Genomics and Integrative Biology, India (Proposal No 45a). All efforts were made to minimize animal suffering.

\section{Zebrafish lines and Maintenance}

Zebrafish line ASWT were bred, raised and maintained at $28.5{ }^{\circ} \mathrm{C}$ according to standard protocols (Westerfield, 2000) and were housed at the CSIR-Institute of Genomics and Integrative Biology (IGIB), Mathura Road New Delhi, India. Embryos were staged both using timing (hours post fertilization (hpf); days post fertilization (dpf)) and morphological features according to (Kimmel et al., 1995). Embryos older than 24hpf were treated with $0.003 \%$ PTU (1-phenyl-2-thiourea) to inhibit pigment formation, aiding fluorescent imaging and RNA in situ hybridization analysis, as and when the experimentation demanded depigmenting the animals. ftyrp 1:GFP plasmid was a kind gift from Dr. Xiangyun Wei (University of Pennsylvania) (Zou et al., 2006) and the construct was injected at a concentration of 10-20 pg alongwith 50-75 pg of Tol2 transposase mRNA into one cell stage zebrafish embryos to create the transgenic line at CSIR-IGIB. Founder lines were established and propagated, which showed expression pattern similar to pt101 as reported in (Zou et al., 2006). The details of zebrafish lines used in this study are provided in key resources table.

\section{Morpholino injections:}

Morpholinos for blocking translational initiation or splicing of RNA were designed by GeneTools $^{\circledR}$ (Supplementary table 1). Minimum effective concentration was determined for each morpholino (MOs) using dosage titration experiments. Concentrations of various MOs used in this study are as follows: 3 - $3.5 \mathrm{ng}$ of $h 2$ a.z. 2 translation block (Z2 MO), 4 - $4.5 \mathrm{ng}$ of h2a.z. 2 splice block (Z2 SB MO), 2 ng of h2a.z. 1 splice block (Z1 MO) (Sivasubbu et al., 2006) and 0.8ng of double targeting (h2a.z.1 and h2a.z.2) translational block (Z1 + Z2 MO). Standard control morpholino from gene tools was used as a control and dosed accordingly. p53 morpholino was co-injected at 1:1 ratio with corresponding gene targeting morpholinos.

\section{Capped RNA injections:}


For rescue experiments, mouse H2a.z. 1 (Z1-RNA) and H2a.z.2 (Z2-RNA) gene were amplified using primers as listed in (Related to STAR methods: Oligonucleotides). Similarly, the zebrafish mitfa gene was amplified using PCR (Related to STAR methods: Oligonucleotides) and cloned into Zero Blunt TOPO vector (Thermo Scientific ${ }^{\circledR}$; K287540) according to manufacturer protocols. Capped and PolyA tail RNA was synthesized by in vitro transcription using T7-Ultra mRNA synthesis kit (Thermo scientific ${ }^{\circledR}$; AM1345) followed by polyA tailing according to manufacturer's protocol. 100pg (for H2a.z.1 and H2a.z.2) or 50pg (for mitfa) was co-injected into 1-cell stage embryos along with the Z2 morpholino.

\section{Whole mount RNA in situ hybridization}

RNA in situ hybridization was performed as described in (Thisse and Thisse, 2008). Riboprobes of various genes foxd3, sox10, crestin, krox20, sox9a (Stewart et al., 2006), mbp (Brosamle and Halpern, 2002), neuroD (van der Velden et al., 2013), isl2a (Appel et al., 1995), sox9b (Yan et al., 2005), tfap2a, mitfa, c-kit, dct (Van Otterloo et al., 2010) were used for this study. Zebrafish h2a.z.2 exon5 - 3'UTR (307 bp) was amplified using primers listed in (Related to STAR methods: Oligonucleotides). The product was subsequently cloned into pCR4-TOPO (Thermo Scientific ${ }^{\circledR} ;$ K287540) and was used as template for in vitro transcription of antisense probe using Megascript T7 (Thermo Scientific ${ }^{\circledR}$, AM1334).

\section{Imaging}

Bright field imaging for live imaging and gene expression pattern (WISH) studies of zebrafish embryos was performed using Zeiss (Stemi 2000C). Zeiss axioscope A1 microscope (with AxiocamHRc) was used for fluorescence imaging. Zeiss proprietary software was used to capture images and processed and analyzed in Adobe Photoshop CS3.

\section{Imaging flow cytometry based population analysis}

Cell counts for sox10:EGFP, mitfa:GFP, ftyrp1:GFP positive cells and ASWT embryos (for melanophore count) were performed using AMNIS ${ }^{\circledR}$ Imaging FACS. Briefly, Embryos were dechorinated using pronase $(5 \mathrm{mg} / \mathrm{ml})$ (Sigma, P8811) for 10-15 mins and collected in microfuge tubes. The embryos were deyolked in ice cold ringer's solution using micropipette tip and spun at $100 \mathrm{~g}$ for 2 minutes at cooled table top centrifuge at $4{ }^{0} \mathrm{C}$ (Eppendorf ${ }^{\circledR}$ centrifuge 5418R). The supernatant was discarded and the embryo bodies were trypsinized using TrypLE Express (Thermoscientific ${ }^{\circledR}, 12604039$ ) for 15 or 30 minutes for $24 \mathrm{hpf}$ or $48 \mathrm{hpf}$ embryos respectively at room temperature. The cell suspension was passed through $70 \mu \mathrm{m}$ cell strainer 
and washed twice with ice cold phosphate buffered saline. The cell suspension was analyzed in the Imaging Flow cytometry system and at least 50,000 - 1,00,000 images were captured and processed using IDEAS (AMNIS ${ }^{\circledR}$ ) software.

\section{Morphant $T g(\operatorname{sox} 10: G F P)$ cell sorting and microarray analysis}

Control MO and Z2 MO were injected in 1 cell stage $\operatorname{Tg}$ (sox 10:GFP) embryos and allowed to develop till $15 \mathrm{hpf}$. The embryos were processed in a similar manner as that for Imaging flow cytometry analysis. Finally, the trypsinised cells were resuspended in ice cold PBS $+10 \%$ Fetal bovine serum and subjected to FACS (BD Bioscience FACSAria ${ }^{\mathrm{TM}}$ III). The GFP positive cells were sorted and collected in RP1 buffer and RNA was isolated using Nucleospin RNA XS kit (Macherey Nagel, 740902) according to manufacturer's protocol. Microarray and analysis was outsourced to Genotypic Technology (Bengaluru, India).

\section{Cell line maintenance and culturing conditions}

B16 mouse melanoma cells were cultured in DMEM-High glucose media (Sigma; D5648) supplemented with 10\% FBS (Thermo Scientific $\left.{ }^{\circledR} ; 10270-106\right)$ at $5 \% \mathrm{CO}_{2}\left(\right.$ Eppendorf $^{\circledR} \mathrm{New}$ Brunswick Galaxy170S). Media was changed every day and cells were passaged upon reaching $80 \%$ confluence. Mouse R1 embryonic stem cells (R1/E) were cultured in DMEM Glutamax media supplemented with sodium pyruvate (Thermo Scientific $\left.{ }^{\circledR} ; 10569-010\right)$, MEM-NEAA (Thermo Scientific $\left.{ }^{\circledR} ; 11140-050\right)$, Beta-mercaptoethanol (Thermo Scientific ${ }^{\circledR} ; 31350-010$ ) and $20 \%$ PANSERA (Pan Biotech; P30-2602) at 5\% $\mathrm{CO}_{2}$. Media was changed every day and cells were passaged every other day.

\section{Melanocyte generation from R1/E mouse embryonic stem cells}

Melanocytes were generated from R1/E mESCs using modified protocol from (Yang et al., 2011). For embryoid body formation, cells were trypsinsed and 50,000 cells/ml suspensions was prepared. 10ul of this suspension was placed in the lid of a $90 \mathrm{~mm}$ petridish and inverted upon the base of the petriplate filled with $15 \mathrm{ml}$ of autoclaved MilliQ. After 4 days the embryoid bodies were transferred to 24 well or 6 well plates coated with collagen (Thermo Scientific ${ }^{\circledR}$; A10483-01) containing Melanocyte conversion media (45\% DMEM - High glucose, 45\% Reconstituted M254 (Thermo Scientific ${ }^{\circledR}$; M254CF), 10\% FBS, 50ng/ml SCF (Peprotech, 300-07-10), 100nM Endothelin-3 (Sigma, E9137), 20pM Cholera toxin (Sigma, C8052), 0.5uM Dexamethasone (Sigma, D1756), 50ng/ml WNT3a (Peprotech; 315-20-10), 4ng/ml beta-FGF (Thermo Scientific ${ }^{\circledR}$; RFGFB50), 50nM PMA (Sigma, P1585) ,1x N2 Supplement 
(Thermo Scientific $\left.{ }^{\circledR} ; 17502-048\right)$ and 1X Anti Anti (Thermo Scientific ${ }^{\circledR} ; 15240-062$ ). Cells were collected at specific time points for downstream analysis.

\section{siRNA and plasmid transfections}

$2 \times 10^{5}$ B16 melanoma cells were seeded in 6 well plates prior to the day of transfection. Next day $100 \mathrm{nM}$ of non targeting control siRNA, H2a.z.2 siRNA (siRNA pool of 5 siRNAs) (Dharmacon $^{\mathrm{TM}}$ ON-TARGETplus; L-063612-01-0005) or H2a.z.1 siRNA (siRNA pool of 5 siRNAs) (Dharmacon ${ }^{\mathrm{TM}}$ ON-TARGETplus; L-042994-01-0005) was transfected according to manufacturer's protocol using Dharmafect (Dharmacon ${ }^{\mathrm{TM}}$, T-2001) transfection reagent. Cells were collected at specified time points for downstream analysis. Plasmid transfections were performed using Lipofectamine 2000 (Thermo Scientific ${ }^{\circledR} ; 11668019$ )

\section{RNA isolation, cDNA synthesis and quantitative Real time PCR}

RNA was isolated from zebrafish embryos or B16 melanoma cells or R1/E cells using Nucleospin Triprep (Macherey Nagel, 740966) according to manufacturer's protocols. cDNA was synthesized using Superscript III First strand cDNA synthesis kit (Thermo Scientific ${ }^{\circledR}$; 1800051). Quantitative real time PCR was carried out as described (Pfaffl, 2001) on ROCHE Lightcycler ${ }^{\circledR}$ Real time PCR system. For quantification, the relative standard curve method was used (as described by the manufacturer) to generate raw values representing arbitrary units of RNA transcripts. Data analysis was performed using $2^{-\Delta \Delta C T}$ method.

\section{shRNA transduction in R1/E ESCs}

Lentiviral packaging and transduction was performed as reported previously by (Motiani et al, 2013a, b). Briefly, lentiviral packaging was done by co-transfecting pVSVG, pdR8.2, and H2a.z. 2 shRNA or Non-targeting shRNA in HEK cells. Two days post-transfections, lentiviral particles were collected from cell supernatant. 1:100 HEPES pH 7.25 1M and 1:100 Polybrene $0.4 \mathrm{mg} / \mathrm{ml}$ was added to lentiviral supernatant. Embryonic stem cell media (ESCM) and lentiviral cocktail were mixed in 1:1 ratio and added to R1/E ESCs. The occulate was spun for $30 \mathrm{~min}$ at $37^{\circ} \mathrm{C}$ and $2500 \mathrm{rpm}$. The cells were incubated at $37^{\circ} \mathrm{C}$ for $10-12$ hours and then media was changed to fresh ESCM. Cells were checked for GFP fluorescence at 48 hours after transduction in $\mathrm{R} 1 / \mathrm{E}$ cells and were selected using $0.5 \mu \mathrm{g} / \mathrm{ml}$ puromycin for a week. Experiments were performed with FACS sorted GFP +ve cells propagated as a pool.

\section{Chromatin Immunoprecipitation and qPCR}


ChIP assays were performed according to protocol provided by Upstate Biotechnology with modifications as suggested in Fast ChIP protocol. ChIP assays were performed using antiH2A.Z antibody (abcam; ab4174). Anti-Rabbit IgG (Thermo Scientific $\left.{ }^{\circledR} ; 02-6102\right)$ was used for isotype control in all the cell lines. Briefly, B16 Melanoma cells or R1/E ESCs were fixed with $10 \%$ formalin (Sigma; HT501128) and incubated at $37^{\circ} \mathrm{C}$ for 10 minutes. $2.5 \mathrm{M}$ Glycine (Sigma; 50046) was added to the cells and again incubated at $37^{\circ} \mathrm{C}$ for 10 minutes. Cells were washed with ice cold 1X PBS containing protease inhibitors. Cells were then scraped and centrifuged at $1000 \mathrm{rpm}$ for 5 minutes at $4^{0} \mathrm{C}$. The cell pellet was lysed in SDS lysis buffer (1\% SDS (Sigma; L3771), 10mM EDTA (Sigma; E6758), 50mM TRIS (Sigma; T6066) (pH 8.1)) on ice for 30 minutes. The cells were then sonicated on bioruptor (DIAGENODE) in ice. The chromatin lysate was then estimated for protein content using BCA kit (Thermo Scientific ${ }^{\circledR}$; 23225). The samples were de-crosslinked overnight at $65^{\circ} \mathrm{C}$, and deproteinised using with Proteinase K (Sigma; P4850). Sheared DNA was column purified using PCR purification kit (QIAGEN; 28104) and estimated using QUBIT dsDNA HS kit (Thermo Scientific ${ }^{\mathbb{R}}$; Q32851). ChIP was performed using 3-5 $\mu$ g of the respective antibody added to equal amount of chromatin across samples and incubated overnight at $4^{\circ} \mathrm{C} .10 \%$ of chromatin was kept separately as input. Next day, 100ul of Protein A agarose beads (G-Bioscience, 786-283) were added to the mixture of chromatin and antibody and incubated for 4-6 hours at $4^{0} \mathrm{C}$. After incubation the beads were washed twice with Low salt Buffer $(0.1 \%$ SDS, $1 \%$ Triton X 100 (Sigma; T8787), 2mM EDTA, 20mM Tris HCl (pH 8), 150mM NaCl (Sigma; S3014)), high salt buffer (0.1\% SDS, $1 \%$ Triton X 100, 2mM EDTA, 20mM Tris $\mathrm{HCl}(\mathrm{pH} 8), 500 \mathrm{mM} \mathrm{NaCl})$ and $\mathrm{LiCl}$ buffer (0.25 M LiCl (Sigma; 62476), 1\% Igepal CA-630 (Sigma; I8896), 1mM EDTA, 10mM Tris $\mathrm{HCl}$ (pH 8), 1\% deoxycholate (Sigma; D6750)). Finally the beads were washed with Tris EDTA buffer. The beads were then incubated with $100 \mu 1$ elution buffer (1\% SDS, $0.75 \%$ sodium bicarbonate (Sigma; S5761)) and $1 \mu 1$ of $20 \mathrm{mg} / \mathrm{ml}$ proteinase $\mathrm{K}$ for 15 minutes; elution was performed again with $100 \mu 1$ for another 15 minutes. Subsequently the samples were kept for $12-14 \mathrm{~h}$ at $65^{\circ} \mathrm{C}$ for reverse crosslinking. After incubation, the samples were column purified using PCR purification kit; the inputs from each of the samples were also included in the purification step. SYBR green (KAPABiosystems; KK4601) based qRT PCR was setup using eluted DNA and graphs were plotted as percentage input. Primer sequence for target genes are provided in Supplementary Table under Oligonucleotide sequences).

\section{Luciferase assay}


Mouse Mitf-luciferase (Kind gift from Dr Krishnamurthy Natarajan, Jawaharal Nehru University) and Sox9-luciferase (Kind gift from Dr Peter Koopman, The University of Queensland) constructs were co-transfected (at ratio of 1:10) with renilla luciferase (pRL-TK vector, PROMEGA) in Non Targeting Control or H2a.z. 2 siRNA treated B16 melanoma cells. After $24 \mathrm{hr}$ the cells were lysed with passive lysis buffer and luciferase assays were performed according to manufacturer's protocols (Dual luciferase reporter assay system; Promega). Alpha MSH (Sigma; M4135) treatment was provided 6 hours post transfection of luciferase constructs.

\section{CRISPR based mutagenesis}

sgRNA targeting zebrafish h2a.z.2 gene were selected from ECRISP (http://www.e-crisp.org/ECRISP/) online tool using default parameters. Primers were designed for generating the complete sgRNA (Related to STAR methods: Oligonucleotides) using annealing PCR. In vitro transcription was performed on these PCR products using T7 Megashortscript kit (Thermo Scientific $^{\circledR}$; AM1354) according to manufacturer's protocols. 100pg of sgRNA was injected with 500 pg of spCAS9 protein (kind gift from Dr Debojyoti Chakraborty, CSIR-IGIB, India). The F0 embryos were grown to adulthood and then were out crossed with ASWT to give rise to F1 animals. In F1 generation, genomic DNA was isolated from fin clips of putative mutants and the target region was amplified using primers provided in (Related to STAR methods: Oligonucleotides). The PCR products were subjected to Sanger sequencing for confirming mutations.

We used the F2 generation h2a.z.2 mutants namely, h2a.z. $2^{\mathrm{Gln} 125 \mathrm{fsX} 144}$, h2a.z. $2^{\text {Ile101fsX110 }}$ and h2a.z. $2^{\Delta 100-128}$ for this study. In the H2a.z. $2^{\mathrm{G} \ln 125 \mathrm{fs} X 144}$ mutants we observe 10 base deletion in exon5, which leads to skipping of the stop codon and adds an additional 14 amino acids to the

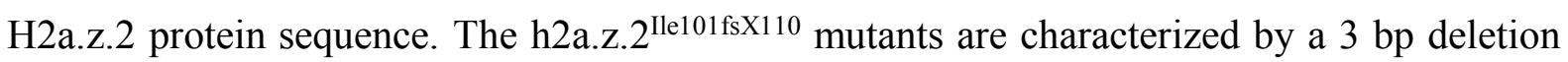
leading to change in amino acid sequences from 100-109 and generating a premature stop codon. The h2a.z. $2^{\Delta 100-128}$ mutants are characterized by 6 base deletion and 3 base insertion leading to premature stop codon at the $100^{\text {th }}$ amino acid.

\section{Melanocyte regeneration via fin amputation}

Fin amputation experiments were performed on heterozygous F1 mutant animals. Mature zebrafish adult (5-10 months) were anesthetized and the distal two-thirds of the caudal fin amputated with a scalpel. The amputated fin was imaged immediately in a stereozoom 
microscope (Zeiss Stemi 2000C). The fish were then returned to fresh water at $25^{\circ} \mathrm{C}$ on a regular feeding schedule for the duration of the experiment. The fin is allowed to regenerate for a week, during which the quiescent MSCs are stimulated and undergo the process of differentiation to generate pigmented cells. The tail fin of the fish was imaged again after 7 days and melanocyte regeneration was assessed.

\section{Statistical analysis and Graphs}

Student's t test was performed to obtain statistical significance in the data. Asterisk on the error bar corresponds to $*(\mathrm{P} \leq 0.05), * *(\mathrm{P} \leq 0.01), * * *(\mathrm{P} \leq 0.001), * * * *(\mathrm{P} \leq 0.0001)$ and $\mathrm{ns}(\mathrm{P}>0.05)$. Graphs were plotted using Graphpad prism.

\section{Acknowledgements}

This work was supported by the Council for Scientific and Industrial Research (CSIR), India through grant (TOUCH-BSC0302, GRAFT-MLP1810 and OLP1118) and Department of Biotechnology through the grant (GAP0182). We acknowledge Dr Chetana Sachidanandan (CSIR-IGIB, New Delhi, India) for providing several zebrafish lines and WISH probes. We acknowledge Dr Aswini Babu for assistance with WISH experiments and Tg (sox10:GFP) embryos FACS sorting. We acknowledge the Imaging and FACS facility of CSIR-IGIB. DAR acknowledges senior research fellowship from ICMR, India.

\section{Author contributions}

D.A.R and T.N.V designed experiments. D.A.R and Y.J.S performed experiments pertaining to zebrafish, D.A.R, Y.J.S, V.G and J.T performed experiments with cultured cells. D.A.R, S.S, R.S.G, R.M and T.N.V were involved in the design and execution of zebrafish experiments. D.A.R, S.S, R.S.G and T.N.V were involved in data analysis, interpretations and writing of the manuscript.

\section{Conflict of interest}

R.S.G. is the co-founder of the board of Vyome Biosciences Pvt Ltd, a biopharmaceutical company in the area of dermatology unrelated to the work presented here. Other authors do not have any competing interests.

\section{Data Availability}

Microarray data of isolated sox10 positive cells is deposited as NCBI GEO data set GSE133141 


\section{References}

1. Adameyko, I., Lallemend, F., Aquino, J. B., Pereira, J. A., Topilko, P., Muller, T., Fritz, N., Beljajeva, A., Mochii, M., Liste, I., et al. (2009). Schwann cell precursors from nerve innervation are a cellular origin of melanocytes in skin. Cell 139, 366-379.

2. Adameyko, I., Lallemend, F., Furlan, A., Zinin, N., Aranda, S., Kitambi, S. S., Blanchart, A., Favaro, R., Nicolis, S., Lubke, M., et al. (2012). Sox2 and Mitf cross-regulatory interactions consolidate progenitor and melanocyte lineages in the cranial neural crest. Development 139, 397-410.

3. Appel, B., Korzh, V., Glasgow, E., Thor, S., Edlund, T., Dawid, I. B. and Eisen, J. S. (1995). Motoneuron fate specification revealed by patterned LIM homeobox gene expression in embryonic zebrafish. Development 121, 4117-4125.

4. Bargaje, R., Alam, M. P., Patowary, A., Sarkar, M., Ali, T., Gupta, S., Garg, M., Singh, M., Purkanti, R., Scaria, V., et al. (2012). Proximity of H2A.Z containing nucleosome to the transcription start site influences gene expression levels in the mammalian liver and brain. Nucleic acids research 40, 8965-8978.

5. Bonisch, C., Schneider, K., Punzeler, S., Wiedemann, S. M., Bielmeier, C., Bocola, M., Eberl, H. C., Kuegel, W., Neumann, J., Kremmer, E., et al. (2012). H2A.Z.2.2 is an alternatively spliced histone H2A.Z variant that causes severe nucleosome destabilization. Nucleic acids research 40, 5951-5964.

6. Brosamle, C. and Halpern, M. E. (2002). Characterization of myelination in the developing zebrafish. Glia 39, 47-57.

7. Cheung, M. and Briscoe, J. (2003). Neural crest development is regulated by the transcription factor Sox9. Development 130, 5681-5693.

8. Chou, W. C., Takeo, M., Rabbani, P., Hu, H., Lee, W., Chung, Y. R., Carucci, J., Overbeek, P. and Ito, M. (2013). Direct migration of follicular melanocyte stem cells to the epidermis after wounding or UVB irradiation is dependent on Mc1r signaling. Nature medicine 19, 924-929.

9. Cox, S. G., Kim, H., Garnett, A. T., Medeiros, D. M., An, W. and Crump, J. G. (2012). An essential role of variant histone $\mathrm{H} 3.3$ for ectomesenchyme potential of the cranial neural crest. PLoS genetics 8, e1002938.

10. Curran, K., Lister, J. A., Kunkel, G. R., Prendergast, A., Parichy, D. M. and Raible, D. W. (2010). Interplay between Foxd3 and Mitf regulates cell fate plasticity in the zebrafish neural crest. Developmental biology 344, 107-118.

11. Dai, X., Bai, Y., Zhao, L., Dou, X., Liu, Y., Wang, L., Li, Y., Li, W., Hui, Y., Huang, X., et al. (2017). H2A.Z Represses Gene Expression by Modulating Promoter Nucleosome Structure and Enhancer Histone Modifications in Arabidopsis. Molecular plant 10, 1274-1292.

12. Donoghue, P. C., Graham, A. and Kelsh, R. N. (2008). The origin and evolution of the neural crest. BioEssays : news and reviews in molecular, cellular and developmental biology 30, 530541.

13. Dooley, C. M., Mongera, A., Walderich, B. and Nusslein-Volhard, C. (2013). On the embryonic origin of adult melanophores: the role of ErbB and Kit signalling in establishing melanophore stem cells in zebrafish. Development 140, 1003-1013.

14. Dryhurst, D., Ishibashi, T., Rose, K. L., Eirin-Lopez, J. M., McDonald, D., Silva-Moreno, B., Veldhoen, N., Helbing, C. C., Hendzel, M. J., Shabanowitz, J., et al. (2009). Characterization of the histone H2A.Z-1 and H2A.Z-2 isoforms in vertebrates. BMC biology 7, 86.

15. Dunn, K. J., Williams, B. O., Li, Y. and Pavan, W. J. (2000). Neural crest-directed gene transfer demonstrates Wnt1 role in melanocyte expansion and differentiation during mouse development. Proceedings of the National Academy of Sciences of the United States of America 97, 10050-10055. 
16. Faast, R., Thonglairoam, V., Schulz, T. C., Beall, J., Wells, J. R., Taylor, H., Matthaei, K., Rathjen, P. D., Tremethick, D. J. and Lyons, I. (2001). Histone variant H2A.Z is required for early mammalian development. Current biology : CB 11, 1183-1187.

17. Gevry, N., Hardy, S., Jacques, P. E., Laflamme, L., Svotelis, A., Robert, F. and Gaudreau, L. (2009). Histone H2A.Z is essential for estrogen receptor signaling. Genes \& development 23, 1522-1533.

18. Goding, C. and Meyskens, F. L., Jr. (2006). Microphthalmic-associated transcription factor integrates melanocyte biology and melanoma progression. Clinical cancer research : an official journal of the American Association for Cancer Research 12, 1069-1073.

19. Goding, C. R. (2000). Mitf from neural crest to melanoma: signal transduction and transcription in the melanocyte lineage. Genes \& development 14, 1712-1728.

20. Hardy, S., Jacques, P. E., Gevry, N., Forest, A., Fortin, M. E., Laflamme, L., Gaudreau, L. and Robert, F. (2009). The euchromatic and heterochromatic landscapes are shaped by antagonizing effects of transcription on H2A.Z deposition. PLoS genetics 5, e1000687.

21. Hu, G., Cui, K., Northrup, D., Liu, C., Wang, C., Tang, Q., Ge, K., Levens, D., Crane-Robinson, C. and Zhao, K. (2013). H2A.Z facilitates access of active and repressive complexes to chromatin in embryonic stem cell self-renewal and differentiation. Cell stem cell 12, 180-192.

22. Hultman, K. A., Budi, E. H., Teasley, D. C., Gottlieb, A. Y., Parichy, D. M. and Johnson, S. L. (2009). Defects in ErbB-dependent establishment of adult melanocyte stem cells reveal independent origins for embryonic and regeneration melanocytes. PLoS genetics 5, e1000544.

23. Ignatius, M. S., Moose, H. E., El-Hodiri, H. M. and Henion, P. D. (2008). colgate/hdac1 Repression of foxd3 expression is required to permit mitfa-dependent melanogenesis. Developmental biology 313, 568-583.

24. Ignatius, M. S., Unal Eroglu, A., Malireddy, S., Gallagher, G., Nambiar, R. M. and Henion, P. D. (2013). Distinct functional and temporal requirements for zebrafish Hdac1 during neural crest-derived craniofacial and peripheral neuron development. PloS one 8, e63218.

25. Iyengar, S., Kasheta, M. and Ceol, C. J. (2015). Poised Regeneration of Zebrafish Melanocytes Involves Direct Differentiation and Concurrent Replenishment of Tissue-Resident Progenitor Cells. Developmental cell 33, 631-643.

26. Jin, E. J., Erickson, C. A., Takada, S. and Burrus, L. W. (2001). Wht and BMP signaling govern lineage segregation of melanocytes in the avian embryo. Developmental biology 233, 22-37.

27. Johnson, S. L., Nguyen, A. N. and Lister, J. A. (2011). mitfa is required at multiple stages of melanocyte differentiation but not to establish the melanocyte stem cell. Developmental biology 350, 405-413.

28. Kaufman, C. K., Mosimann, C., Fan, Z. P., Yang, S., Thomas, A. J., Ablain, J., Tan, J. L., Fogley, R. D., van Rooijen, E., Hagedorn, E. J., et al. (2016). A zebrafish melanoma model reveals emergence of neural crest identity during melanoma initiation. Science 351, aad2197.

29. Kawakami, A. and Fisher, D. E. (2011). Key discoveries in melanocyte development. The Journal of investigative dermatology 131, E2-4.

30. ---- (2017). The master role of microphthalmia-associated transcription factor in melanocyte and melanoma biology. Laboratory investigation; a journal of technical methods and pathology 97, 649-656.

31. Kimmel, C. B., Ballard, W. W., Kimmel, S. R., Ullmann, B. and Schilling, T. F. (1995). Stages of embryonic development of the zebrafish. Developmental dynamics : an official publication of the American Association of Anatomists 203, 253-310.

32. Kos, R., Reedy, M. V., Johnson, R. L. and Erickson, C. A. (2001). The winged-helix transcription factor FoxD3 is important for establishing the neural crest lineage and repressing melanogenesis in avian embryos. Development 128, 1467-1479.

33. Le Douarin, N. M., Creuzet, S., Couly, G. and Dupin, E. (2004). Neural crest cell plasticity and its limits. Development 131, 4637-4650. 
34. Le Douarin, N. M. and Dupin, E. (2003). Multipotentiality of the neural crest. Current opinion in genetics \& development 13, 529-536.

35. Lister, J. A., Robertson, C. P., Lepage, T., Johnson, S. L. and Raible, D. W. (1999). nacre encodes a zebrafish microphthalmia-related protein that regulates neural-crest-derived pigment cell fate. Development 126, 3757-3767.

36. Madakashira, B., Corbett, L., Zhang, C., Paoli, P., Casement, J. W., Mann, J., Sadler, K. C. and Mann, D. A. (2017). Variant Histone H2afv reprograms DNA methylation during early zebrafish development. Epigenetics 12, 811-824.

37. Marathe, H. G., Watkins-Chow, D. E., Weider, M., Hoffmann, A., Mehta, G., Trivedi, A., Aras, S., Basuroy, T., Mehrotra, A., Bennett, D. C., et al. (2017). BRG1 interacts with SOX10 to establish the melanocyte lineage and to promote differentiation. Nucleic acids research $\mathbf{4 5}$, 6442-6458.

38. Martik, M. L. and Bronner, M. E. (2017). Regulatory Logic Underlying Diversification of the Neural Crest. Trends in genetics : TIG 33, 715-727.

39. Matsuda, R., Hori, T., Kitamura, H., Takeuchi, K., Fukagawa, T. and Harata, M. (2010). Identification and characterization of the two isoforms of the vertebrate H2A.Z histone variant. Nucleic acids research 38, 4263-4273.

40. Murphy, P. J., Wu, S. F., James, C. R., Wike, C. L. and Cairns, B. R. (2018). Placeholder Nucleosomes Underlie Germline-to-Embryo DNA Methylation Reprogramming. Cell 172, 9931006 e1013.

41. Nishimura, E. K., Jordan, S. A., Oshima, H., Yoshida, H., Osawa, M., Moriyama, M., Jackson, I. J., Barrandon, Y., Miyachi, Y. and Nishikawa, S. (2002). Dominant role of the niche in melanocyte stem-cell fate determination. Nature 416, 854-860.

42. Pfaffl, M. W. (2001). A new mathematical model for relative quantification in real-time RTPCR. Nucleic acids research 29, e45.

43. Punzeler, S., Link, S., Wagner, G., Keilhauer, E. C., Kronbeck, N., Spitzer, R. M., Leidescher, S., Markaki, Y., Mentele, E., Regnard, C., et al. (2017). Multivalent binding of PWWP2A to H2A.Z regulates mitosis and neural crest differentiation. The EMBO journal 36, 2263-2279.

44. Rai, K., Jafri, I. F., Chidester, S., James, S. R., Karpf, A. R., Cairns, B. R. and Jones, D. A. (2010). Dnmt3 and G9a cooperate for tissue-specific development in zebrafish. The Journal of biological chemistry 285, 4110-4121.

45. Raible, D. W. and Eisen, J. S. (1994). Restriction of neural crest cell fate in the trunk of the embryonic zebrafish. Development 120, 495-503.

46. Sivasubbu, S., Balciunas, D., Davidson, A. E., Pickart, M. A., Hermanson, S. B., Wangensteen, K. J., Wolbrink, D. C. and Ekker, S. C. (2006). Gene-breaking transposon mutagenesis reveals an essential role for histone H2afza in zebrafish larval development. Mechanisms of development 123, 513-529.

47. Soldatov, R., Kaucka, M., Kastriti, M. E., Petersen, J., Chontorotzea, T., Englmaier, L., Akkuratova, N., Yang, Y., Haring, M., Dyachuk, V., et al. (2019). Spatiotemporal structure of cell fate decisions in murine neural crest. Science $\mathbf{3 6 4}$.

48. Stewart, R. A., Arduini, B. L., Berghmans, S., George, R. E., Kanki, J. P., Henion, P. D. and Look, A. T. (2006). Zebrafish foxd3 is selectively required for neural crest specification, migration and survival. Developmental biology 292, 174-188.

49. Takeda, K., Yasumoto, K., Takada, R., Takada, S., Watanabe, K., Udono, T., Saito, H., Takahashi, K. and Shibahara, S. (2000). Induction of melanocyte-specific microphthalmiaassociated transcription factor by Wnt-3a. The Journal of biological chemistry 275, 1401314016.

50. Thisse, C. and Thisse, B. (2008). High-resolution in situ hybridization to whole-mount zebrafish embryos. Nature protocols 3, 59-69. 
51. van der Velden, Y. U., Wang, L., Querol Cano, L. and Haramis, A. P. (2013). The polycomb group protein ring1b/rnf2 is specifically required for craniofacial development. PloS one $\mathbf{8}$, e73997.

52. Van Otterloo, E., Li, W., Bonde, G., Day, K. M., Hsu, M. Y. and Cornell, R. A. (2010). Differentiation of zebrafish melanophores depends on transcription factors AP2 alpha and AP2 epsilon. PLoS genetics 6, e1001122.

53. Vardabasso, C., Gaspar-Maia, A., Hasson, D., Punzeler, S., Valle-Garcia, D., Straub, T., Keilhauer, E. C., Strub, T., Dong, J., Panda, T., et al. (2015). Histone Variant H2A.Z.2 Mediates Proliferation and Drug Sensitivity of Malignant Melanoma. Molecular cell 59, 75-88.

54. Wagner, D. E., Weinreb, C., Collins, Z. M., Briggs, J. A., Megason, S. G. and Klein, A. M. (2018). Single-cell mapping of gene expression landscapes and lineage in the zebrafish embryo. Science 360, 981-987.

55. Watanabe, A., Takeda, K., Ploplis, B. and Tachibana, M. (1998). Epistatic relationship between Waardenburg syndrome genes MITF and PAX3. Nature genetics 18, 283-286.

56. Westerfield, M. (2000). A guide for the laboratory use of Zebrafish (Danio rerio). Univ. of Oregon Press. Eugene.

57. White, R. M. and Zon, L. I. (2008). Melanocytes in development, regeneration, and cancer. Cell stem cell 3, 242-252.

58. Wratting, D., Thistlethwaite, A., Harris, M., Zeef, L. A. and Millar, C. B. (2012). A conserved function for the H2A.Z C terminus. The Journal of biological chemistry 287, 19148-19157.

59. Yan, Y. L., Willoughby, J., Liu, D., Crump, J. G., Wilson, C., Miller, C. T., Singer, A., Kimmel, C., Westerfield, M. and Postlethwait, J. H. (2005). A pair of Sox: distinct and overlapping functions of zebrafish sox9 co-orthologs in craniofacial and pectoral fin development. Development 132, 1069-1083.

60. Yang, R., Jiang, M., Kumar, S. M., Xu, T., Wang, F., Xiang, L. and Xu, X. (2011). Generation of melanocytes from induced pluripotent stem cells. The Journal of investigative dermatology 131, 2458-2466.

61. Zou, J., Beermann, F., Wang, J., Kawakami, K. and Wei, X. (2006). The Fugu tyrp1 promoter directs specific GFP expression in zebrafish: tools to study the RPE and the neural crest-derived melanophores. Pigment cell research 19, 615-627.

62. Zovkic, I. B., Paulukaitis, B. S., Day, J. J., Etikala, D. M. and Sweatt, J. D. (2014). Histone H2A.Z subunit exchange controls consolidation of recent and remote memory. Nature 515, 582-586. 
Figure Legends:

\section{Figure 1: $H 2 a . z .2$ controls the melanocyte numbers during zebrafish development}

(A) Bright field images of control morpholino (control MO) and H2a.z.2 morpholino injected embryos (Z2 MO) at 2 days post fertilization (dpf).

(B) Fluorescence image of $T g(f t y r p 1: G F P)$ that tags differentiating melanophores, in control and $\mathrm{Z2} \mathrm{MO}$ embryos at $2 \mathrm{dpf}$.

(C) Bright field images of control and Z2 MO at $7 \mathrm{dpf}$. (inset) Enlarged view of the lateral line melanophores.

(D) Western blot analysis of control and Z2 MO embyos at 2 dpf, carried out using an antibody that recognizes both H2A.Z.1 and H2A.Z.2 proteins.

(E) Bar graphs represent means \pm SEM of percent embryos with varying degree of pigmentation (depigmented, partially pigmented and normally pigmented) scored manually at 2dpf, from embryos injected with control MO, Z2 MO, Z2 MO along with mouse H2a.z.1 mRNA or mouse H2a.z. 2 mRNA.

(F) Inverted bar graphs represent mean $\pm \operatorname{SEM}(n=3)$ in the percent reduction in cell numbers of Z2 MO compared to control in various marker lines. Time of assessment of labelled cells, their identity and the transgenic line used are indicated.

(G) Whole mount RNA in situ hybridization (WISH) based expression pattern of early neural crest markers foxd 3 , sox 10 and tfap $2 a$ at $11 \mathrm{hpf}$.

(H) WISH based expression pattern of neural crest markers crestin, sox 10 and foxd 3 at $24 \mathrm{hpf}$. Numbers in WISH images indicate frequency of the represented phenotype in total number of embryos analyzed. Scale bars $100 \mu \mathrm{m}$.

\section{Figure 2: H2a.z.2 alters neural crest gene regulatory network, and decreases melanocyte and glial footprint}

(A) Schematic design of microarray experiment.

(B and C) Bubble plots representing gene ontology functions of differentially regulated genes with a $\log _{2}$ fold change $\leq-0.6$ (downregulated, B) and $\geq 0.6$ (upregulated, C) upon silencing $\mathrm{Z} 2$ in sox $10+$ ve cells.

(D) Heatmaps representing differential expression of key transcription factors in sox $10+v e$ cells of Z2 MO as compared to control across melanocyte, glial and neuronal lineages.

(E) WISH based expression pattern of melanocyte markers ckit and $d c t$ at $24 \mathrm{hpf}$

(F) $\mathrm{Tg}$ (foxd3:GFP) labelling of glial cells that mark undifferentiated glia and (bottom) WISH of glial markers krox20 and $m b p$ at $5 \mathrm{dpf}$.

(G) $\operatorname{Tg}(n b:$ dsRed) labelling of neurons in the spinal chord bundle (lateral view). (Bottom) WISH isl2a marker for cranial motor neurons.

(H) $\operatorname{Tg}($ mitfa:GFP) labelling of melanocytes, (bottom) WISH of mitfa at $24 \mathrm{hpf}$.

(I) Bright field images of control, Z2 MO and Z2 MO coinjected with mitfa mRNA at $48 \mathrm{hpf}$.

(J) Bars represent mean \pm SEM $(n=3)$ of the number of head melanophores with at least 30 embryos each.

(K) Schematic representation of the neural crest derived lineages, highlighting the dependence of melanocyte and glial cells on h2a.z.2

Numbers in WISH images indicate frequency of the represented phenotype in total number of embryos analyzed. Scale bars represent $100 \mu \mathrm{m}$.

\section{Figure 3: H2.a.z.2 controls melanocyte derivation from mouse embryonic stem cells in vitro}

(A) Schematic of melanocyte derivation from mouse R1/E stem cells.

(B) Kinetics of expression patterns of stemness genes (Oct4 and Nanog); pigmentation genes

(Mitf and Dct); neural crest genes (Sox9 and Sox10) along with H2a.z.1 and H2a.z.2 at

different stages of melanocyte derivation from mouse R1/E stem cells. 
(C) Bright field and GFP images of embryoid bodies derived from R1/E cells silenced using lentiviral contruct for non-targetting or Z2 shRNA, encoding GFP marker.

(D) Bar graphs representing qRT-PCR for H2.a.z.1 (blue) and H2.a.z.2 (red) in shZ2 cells compared to shNT at the embryoid body stage.

(E-G) Bar graphs representing qRT-PCR analysis for Sox10, Mitf and Tyr (tyrosinase) on 6 days post induction of differentiation.

(H) Heatmap representing the promoter occupancy of H2.A.Z (H2.A.Z.1 and H2.A.Z.2) determined by Chromatin Immunoprecipitation (ChIP) and the corresponding mRNA levels of Mitf, Sox9, Sox10 and Ckit during various stages of melanocyte derivation from R1/E cells. Numbers in the heat map represent percent enrichment of H2A.Z occupancy in ChIP and fold change compared to R1/E cells in the quantitative real-time PCR for the mRNA levels. (I) H2A.Z occupancy at the indicated promoters in melanocytes (depigmented B16 cells) is represented as a heat map of percent input for H2.A.Z as well as normal rabbit IgG.

Numbers in the heat map represent percent enrichment of the promoter DNA in H2A.Z or IgG ChIP.

(J,L,N) H2A.Z ChIP of Sox10, Sox9 and Mitf promoters upon Z2 knockdown.

$(\mathrm{K}, \mathrm{M}, \mathrm{O})$ mRNA levels of Sox10, Sox9 and Mitf upon Z2 knockdown.

(P) Western blot for H2a.Z upon Z2 overexpression.

(Q) Reporter assays for Mitf promoter by dual luciferase assay in control and H2.a.z.2

overexpressed cells in basal as well as $\alpha$-Melanocyte Stimulating Hormone $(\alpha-\mathrm{MSH})$ treated

B16 cells for $24 \mathrm{~h}$. Bar graphs represent mean $\pm \operatorname{SEM}(\mathrm{n}=3)$.

(R) Schematic representation of the role of H2.a.z. 2 in modulating Mitf promoter.

Figure 4: Targeted mutation of $h 2 a . z .2$ affects melanocyte specification and regeneration

(A) Orthology based schematic representation of the secondary structure of zebrafish H2A.Z.2. The functional elements in the protein are denoted above.(below) Sequence of the amino acids in H2A.Z.2, its variant H2A.Z.2.2 and the three CRISPR mutants generated in this study.

(B) Representative images of wild type and Z2 mutant adult fishes. (right) zoomed in images of first ventral stripe. (Adult fishes were imaged in parts and stitched)

(D) Fin clip and regeneration images of adult wild type and Z2 mutant animals. Right panel represents fins after 7 days of regeneration.

\section{Expanded View Figure Legends}

\section{Suppl Fig 1: Validating H2a.z.2 specific pigmentation phenotype using multiple silencing approaches}

(A) Schematic representing the target region for H2a.z.2 MO (Z.2), H2a.z.1 MO (Z.1) and Z.2 \& Z.1 MO.

(B) Brightfield images representing 48 hpf embryos injected with Control MO, Z.2 MO, Z.1 MO, Z.1\& Z.2 MO.

(C) Brightfield images showing dorsal and lateral view of Control MO, Z.2 MO and Z.2 SB MO embryos at $48 \mathrm{hpf}$.

(D) H2a.z.2 RT-PCR amplicons from control and Z.2 SB MO injected embryos depicting the mis-spliced product.

(E) Bar graphs representing relative p53 mRNA levels in Z.2 MO as compared to control MO.

(F) Grouped bar plots representing percentage survival and pigmentation phenotype observed across control MO, Z2 MO and Z.2+ p53 MO.

Suppl Fig 2: Status of neural crest derivatives in H2a.z.2 morphants 
(A) Table enumerating the number of enteric, and trunk ectopic neurons observed in control and Z.2 morphants. Numbers represent mean \pm SD counted manually across $\sim 50$ embryos.

(B) Fluorescence images of enteric neurons in control and Z.2 morphants.

(C) Alcian blue staining highlighting the craniofacial cartilage and fin cartilage in control and Z.2 morphants.

(D) Fluorescence images of $T g(\operatorname{sox} 10: E G F P)$ head region representing the craniofacial cartilage system in control and Z.2 morphants.

\section{Suppl Fig 3: H2A.Z occupancy and gene expression changes.}

(A-C) Metanalysis of chromatin immunoprecipitaton (ChIP) data in melanocyte derived lines from GSE68223.

(A) H2A.Z (Z.1 + Z.2) occupancy near transcription start site (TSS) in primary human melanocytes.

(B) H2A.Z.2-GFP occupancy near TSS in SK-Mel 147 metastatic melanoma cells.

(C) H2A.Z.1-GFP occupancy near TSS in SK-Mel 147 metastatic melanoma cells. Dotted line indicates the TSS. Gene names are displayed on right corresponding to their ChIP seq profiles. Percent enrichment relative to input in the ChIP-seq data is displayed to the left.

(D) Bar graph representing mRNA levels of H2a.z.1 and H2a.z.2 upon Z.1 silencing (mean \pm SEM, N=3).

(E) Bar graph representing mRNA levels of H2a.z.1 and H2a.z.2 upon Z.2 silencing (mean \pm SEM, N=3).

(F) Bar graphs representing the relative Sox9 luciferase activity of cells treated with non targeting control and Z.2 siRNA (mean $\pm \mathrm{SEM}, \mathrm{N}=3$ ).

(G) Bar plot representing relative mRNA levels of pigmentation related genes upon Z.2 and Z.1 silencing in $\mathrm{B} 16$ cells (mean $\pm \mathrm{SEM}, \mathrm{N}=2$ ).

\section{Suppl Fig 4: Targeted knockout of $h 2 a_{.} z_{0} 2$ is lethal in zebrafish}

(A) Schematic representing the region targeted by h2a.z. 2 sgRNA1.

(B) Sequences displaying representative mutations occurred upon h2a.z.2 sgRNA1 injections leading to embryonic lethality; predicted protein sequences are displayed on the right hand side.

(C) Bright field images of SpCas9 and SpCas9 + sgRNA1 injected embryos at 6 and $36 \mathrm{hpf}$. 


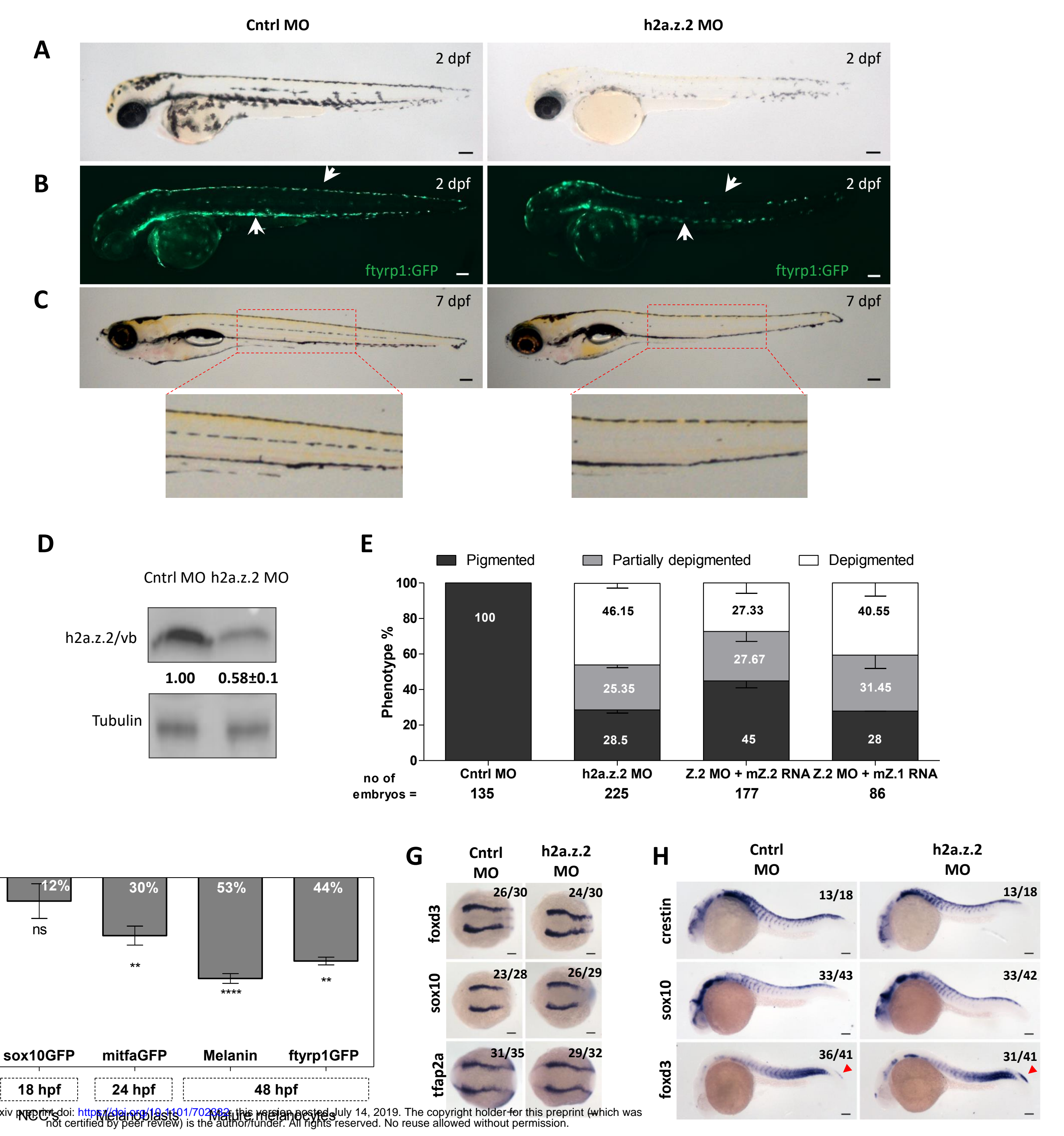

FIG 1 
A

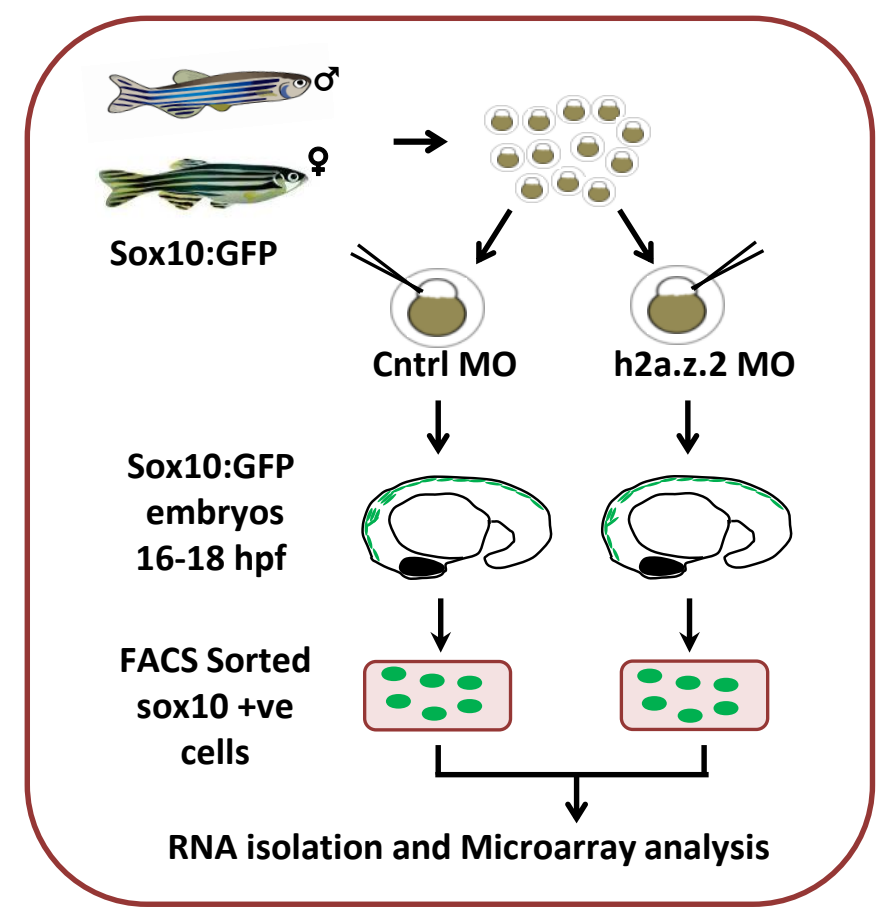

C

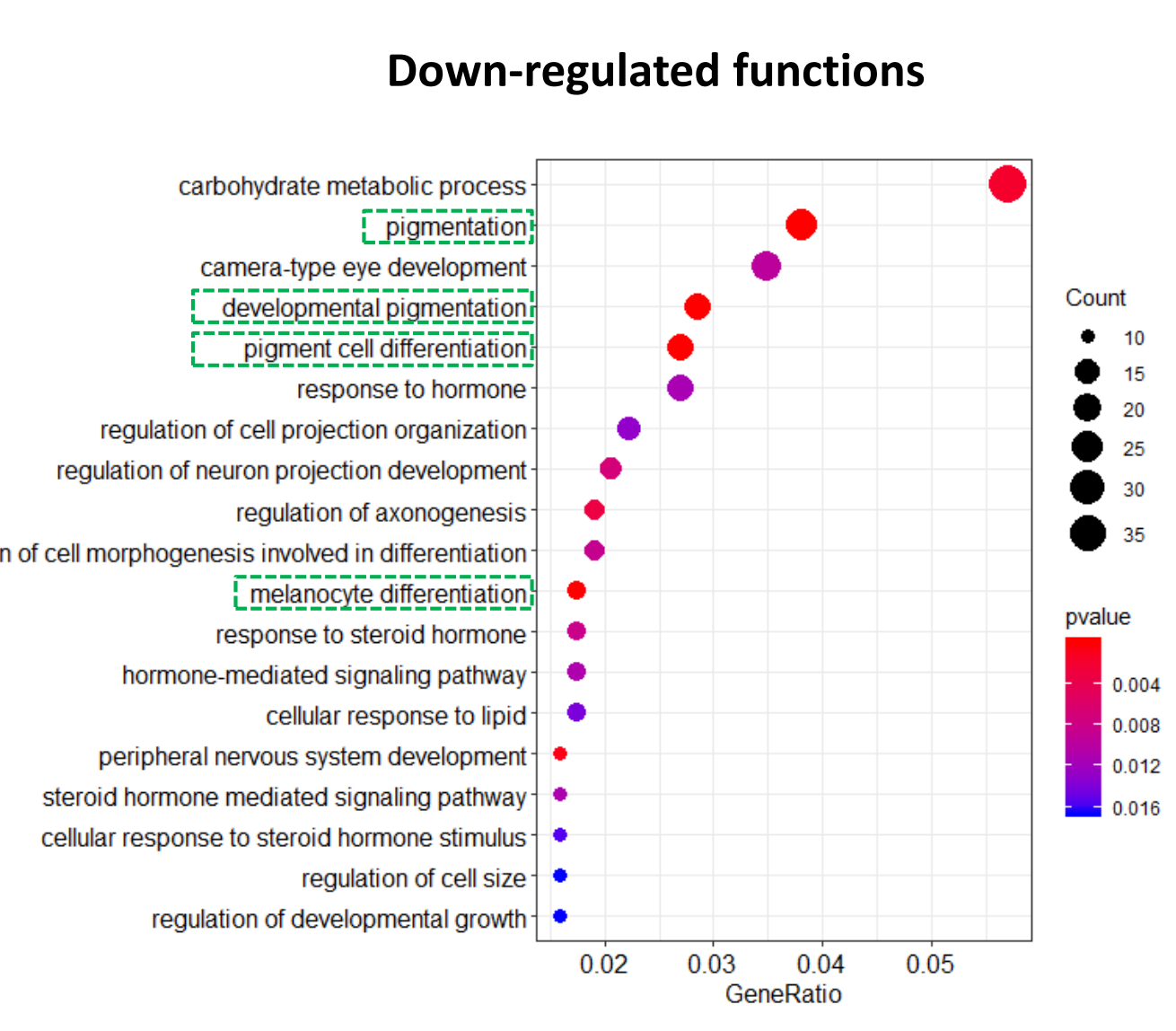

E

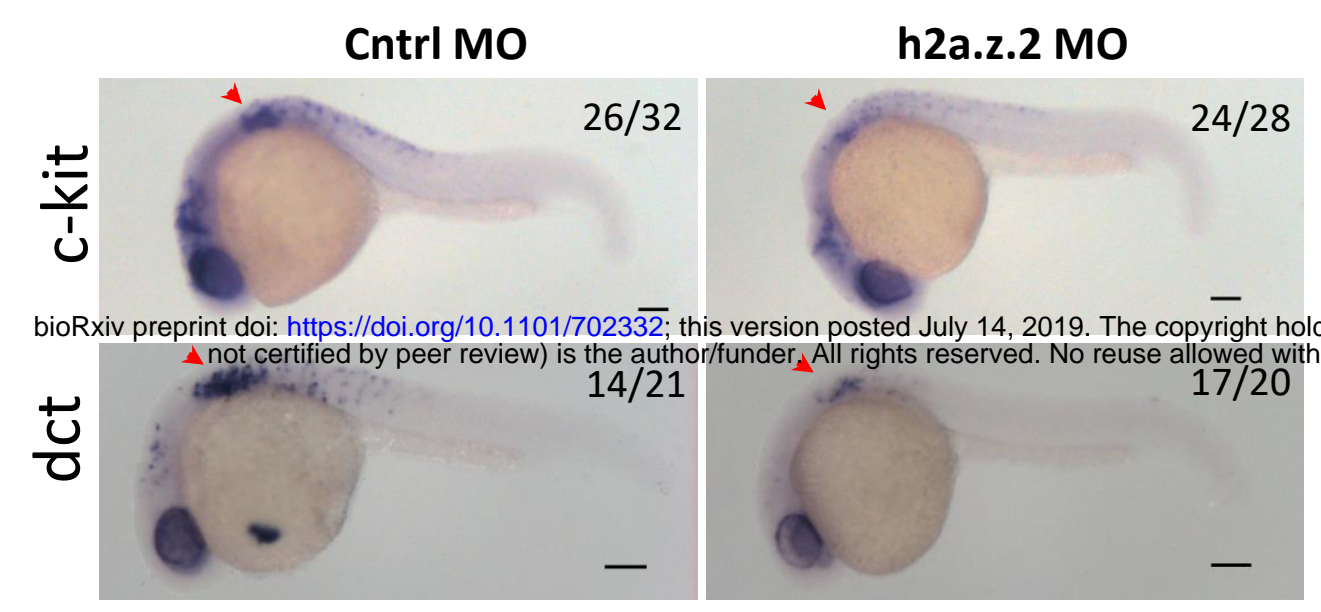

G

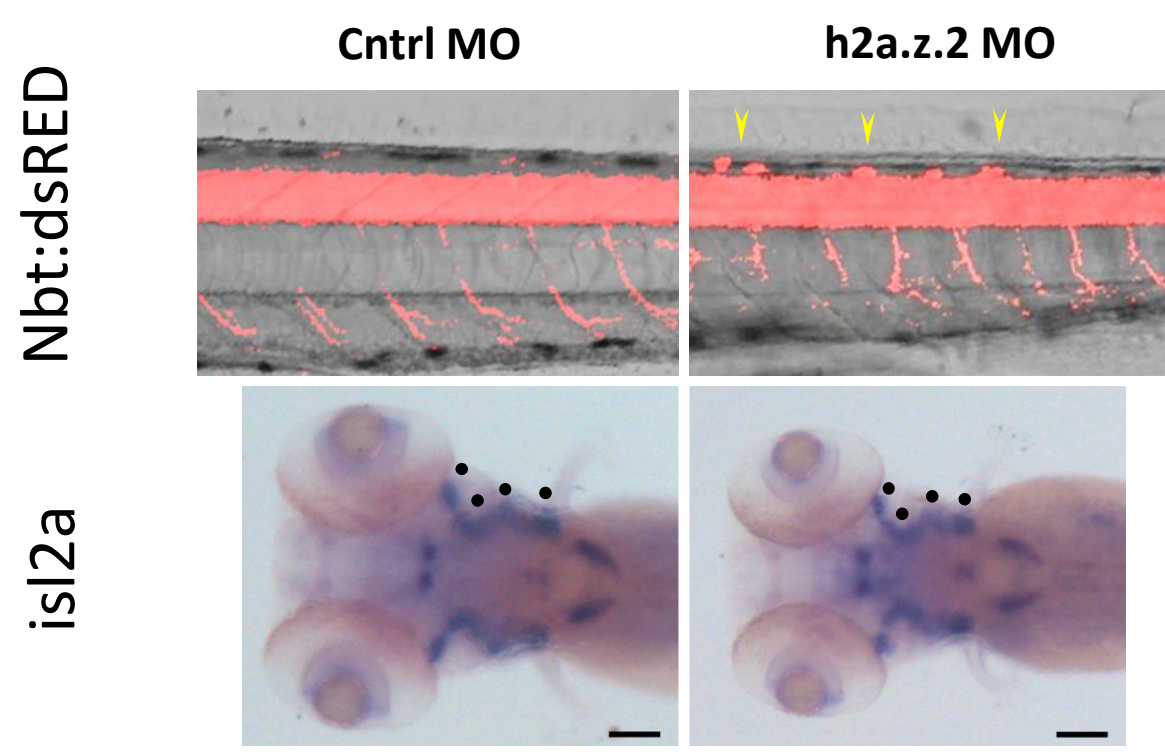

H
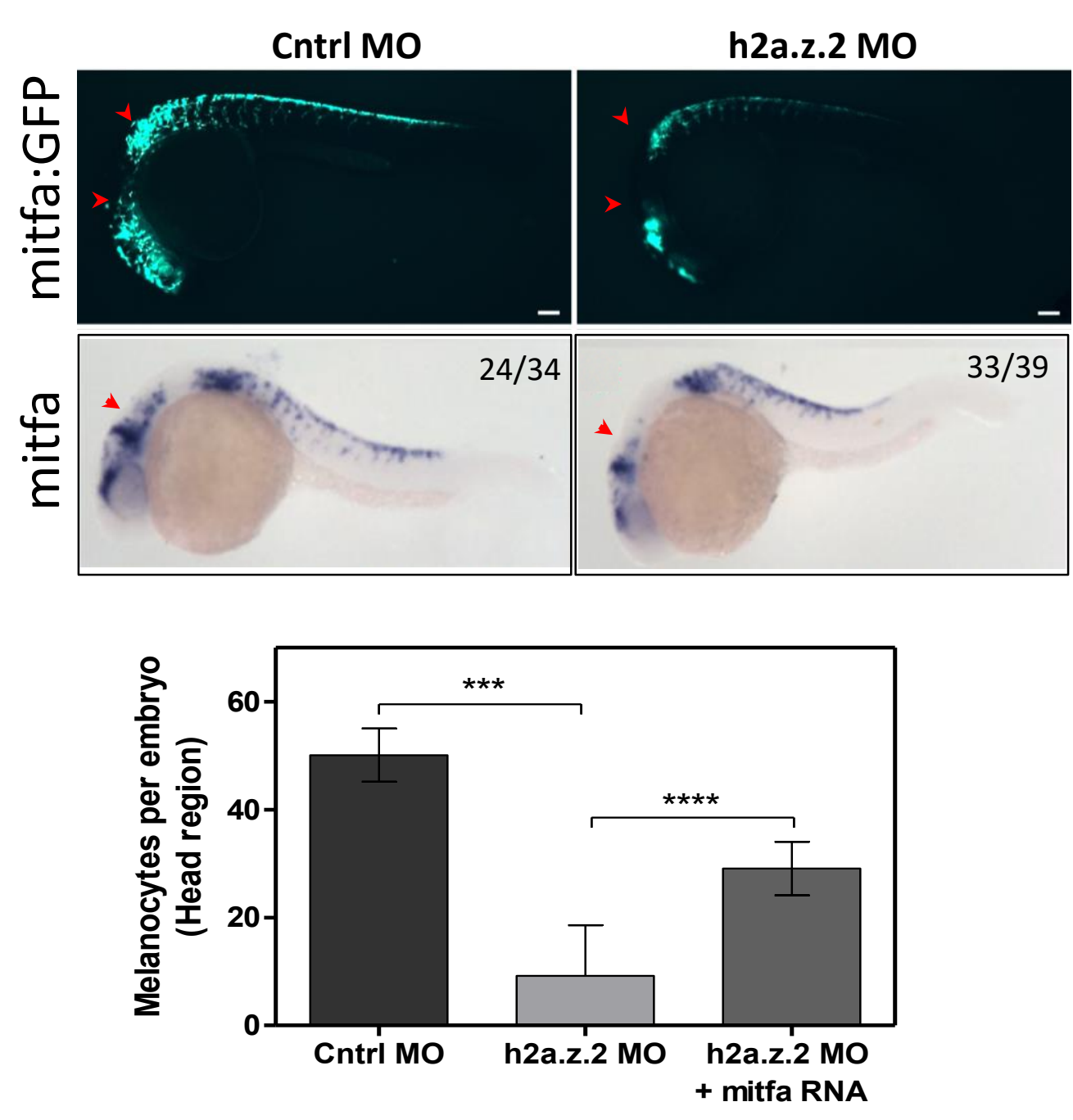

B

Up-regulated functions
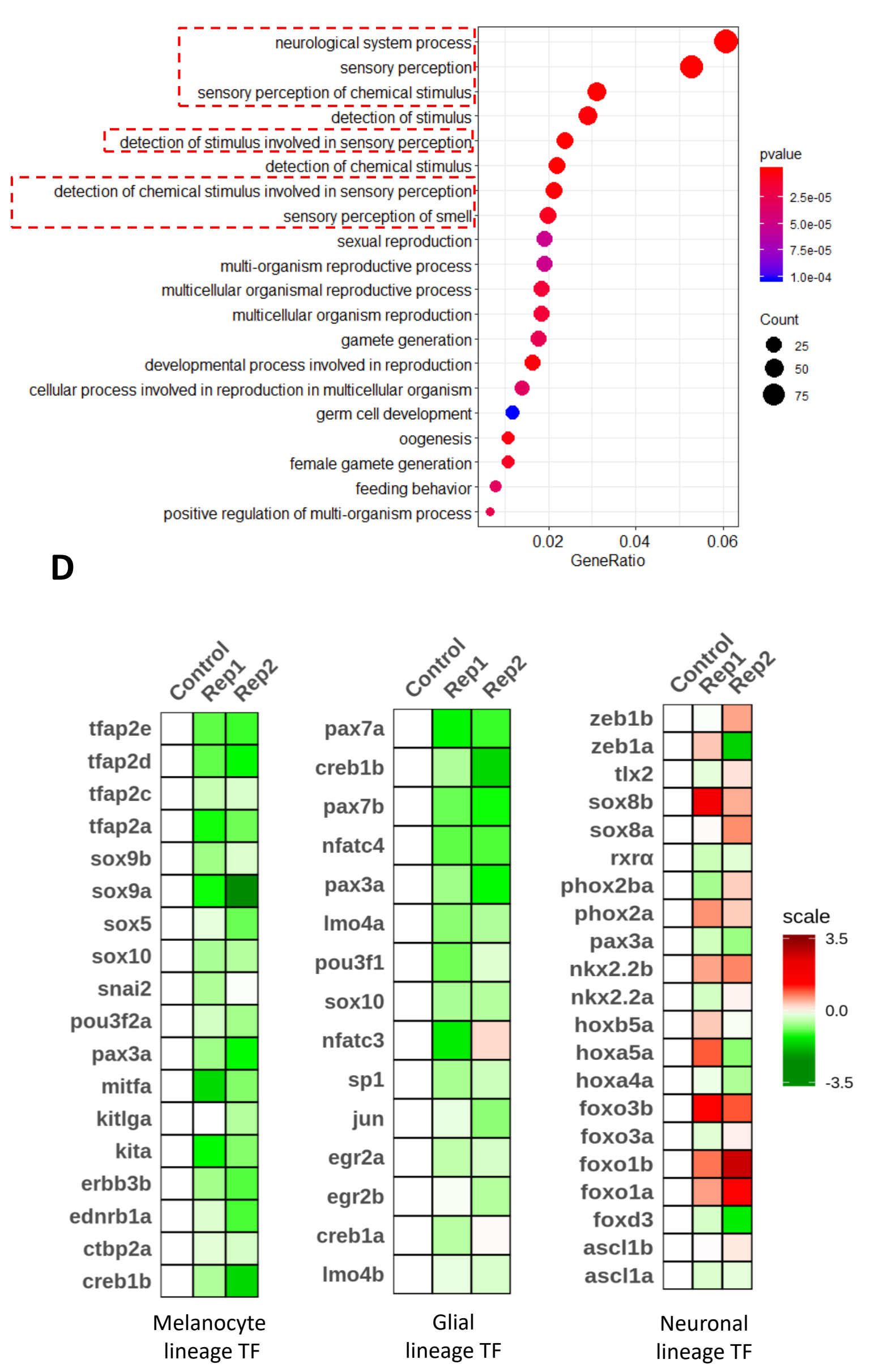

F

Cntrl MO

h2a.z.2 MO
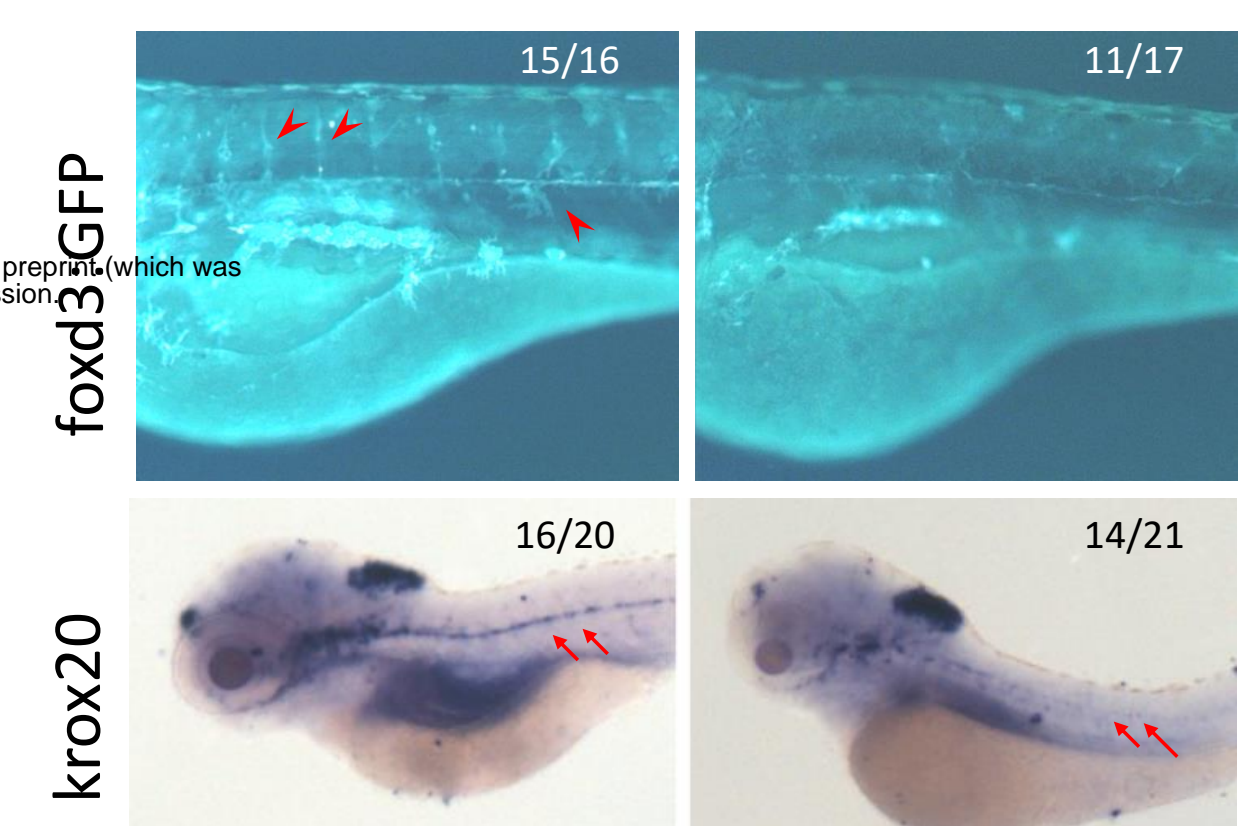

$14 / 21$
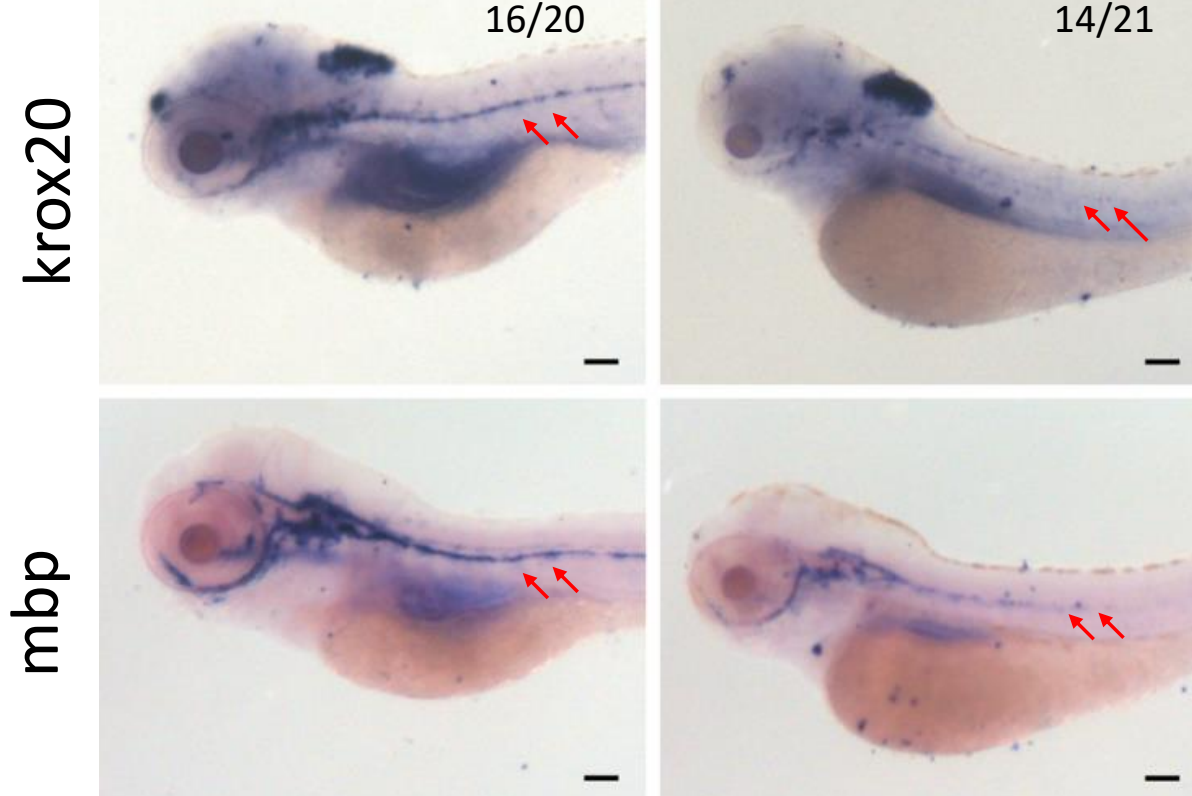

I

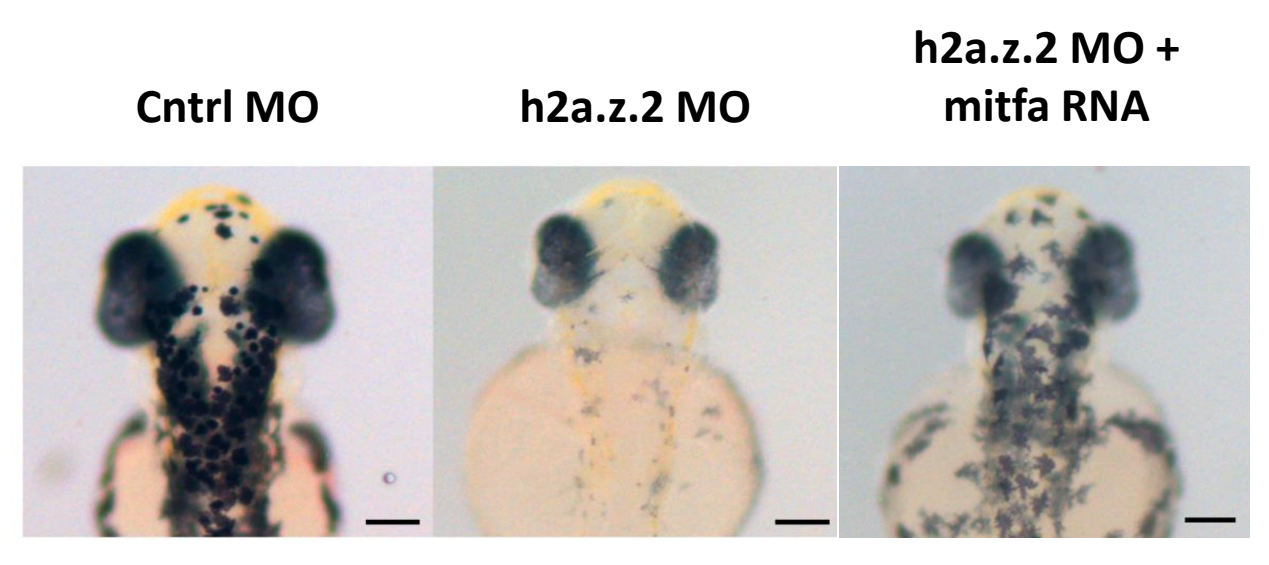

K

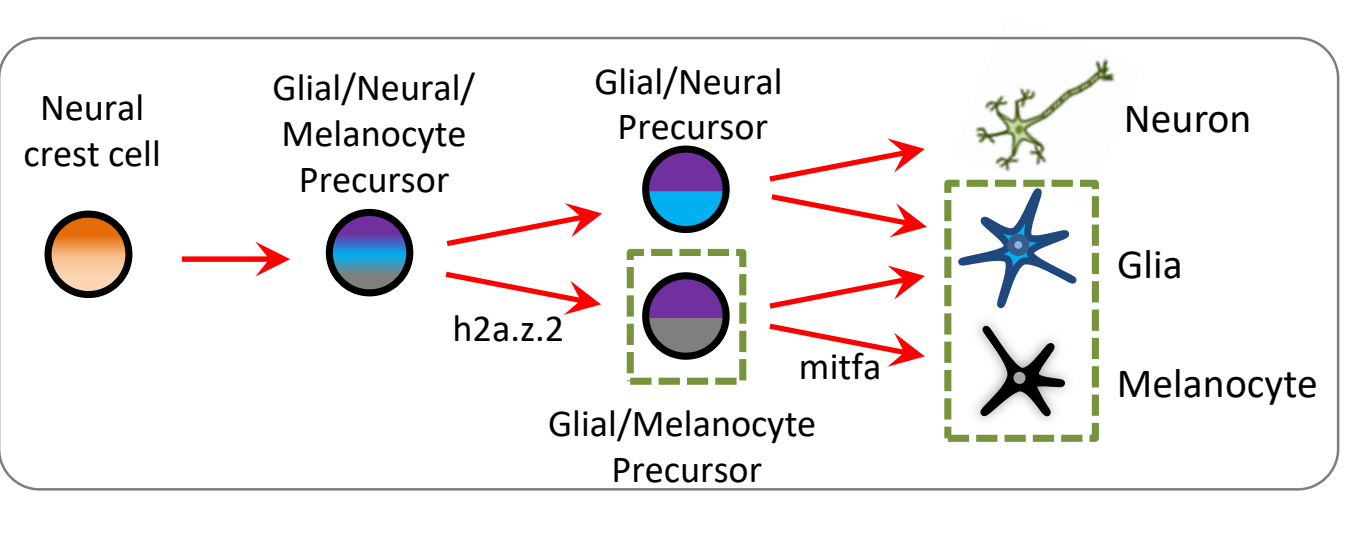


A

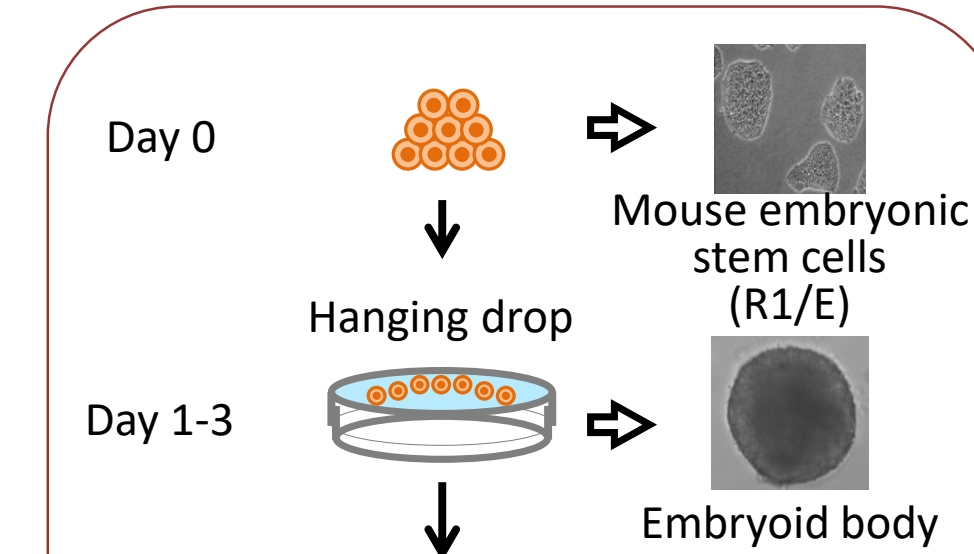

Melanocyte specification (EB)

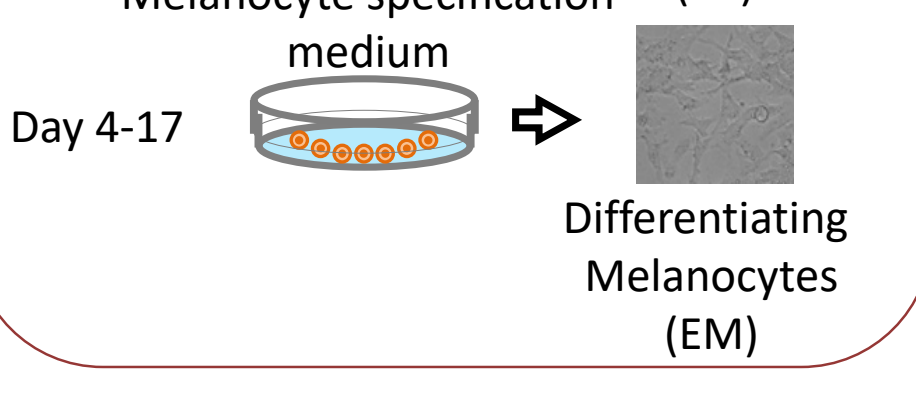

C

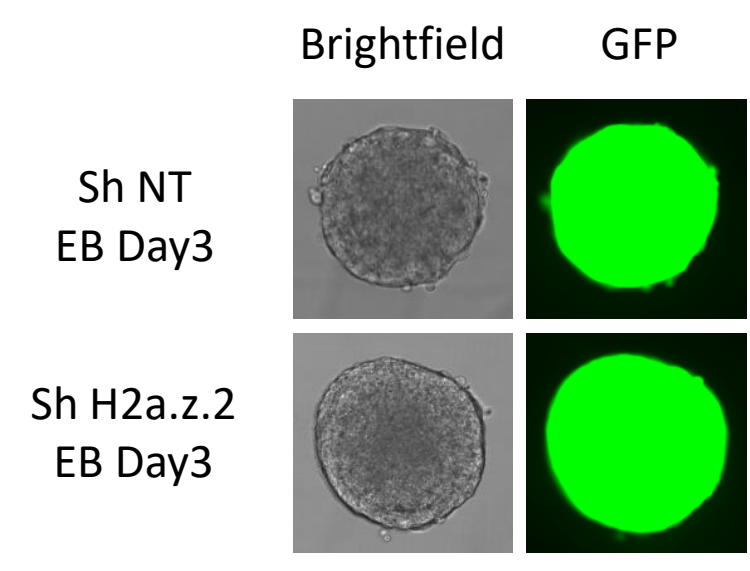

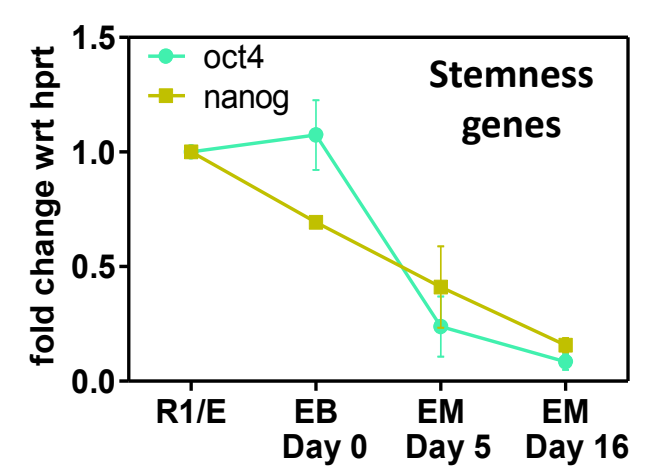
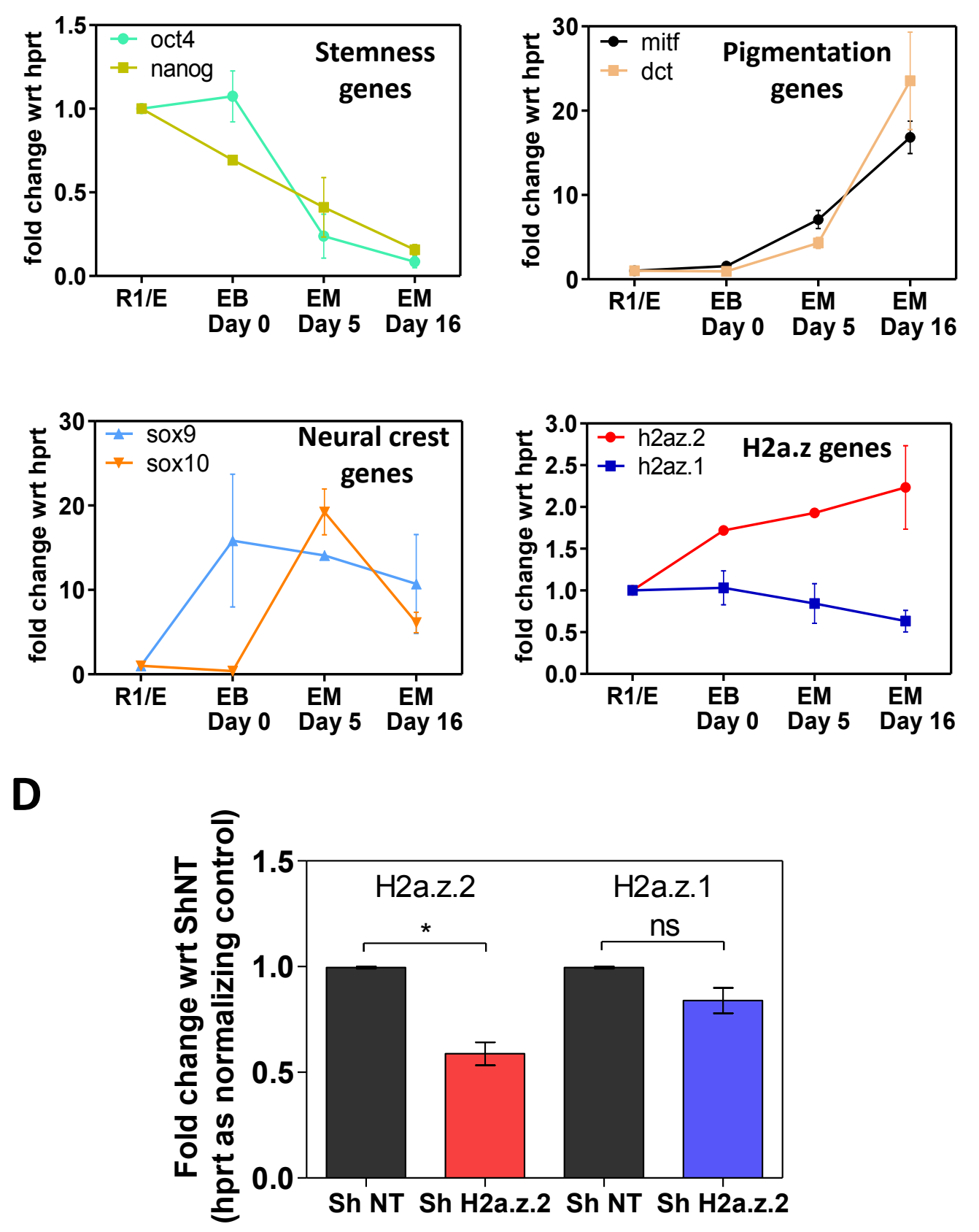

E

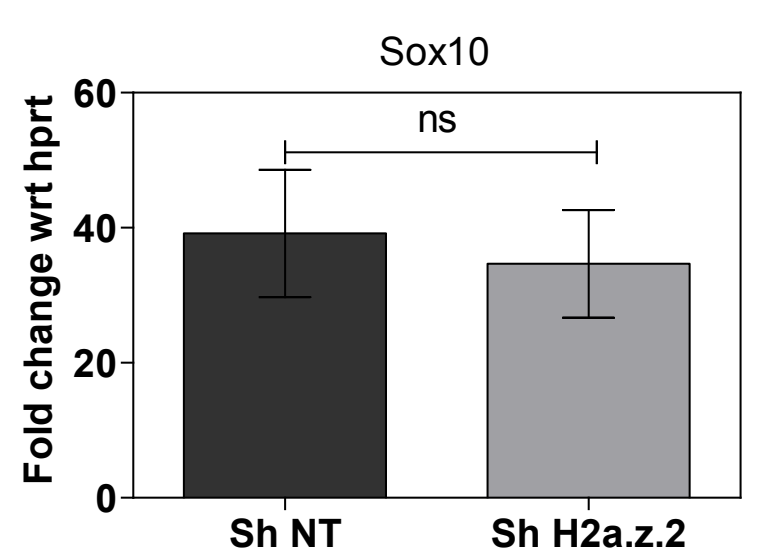

H
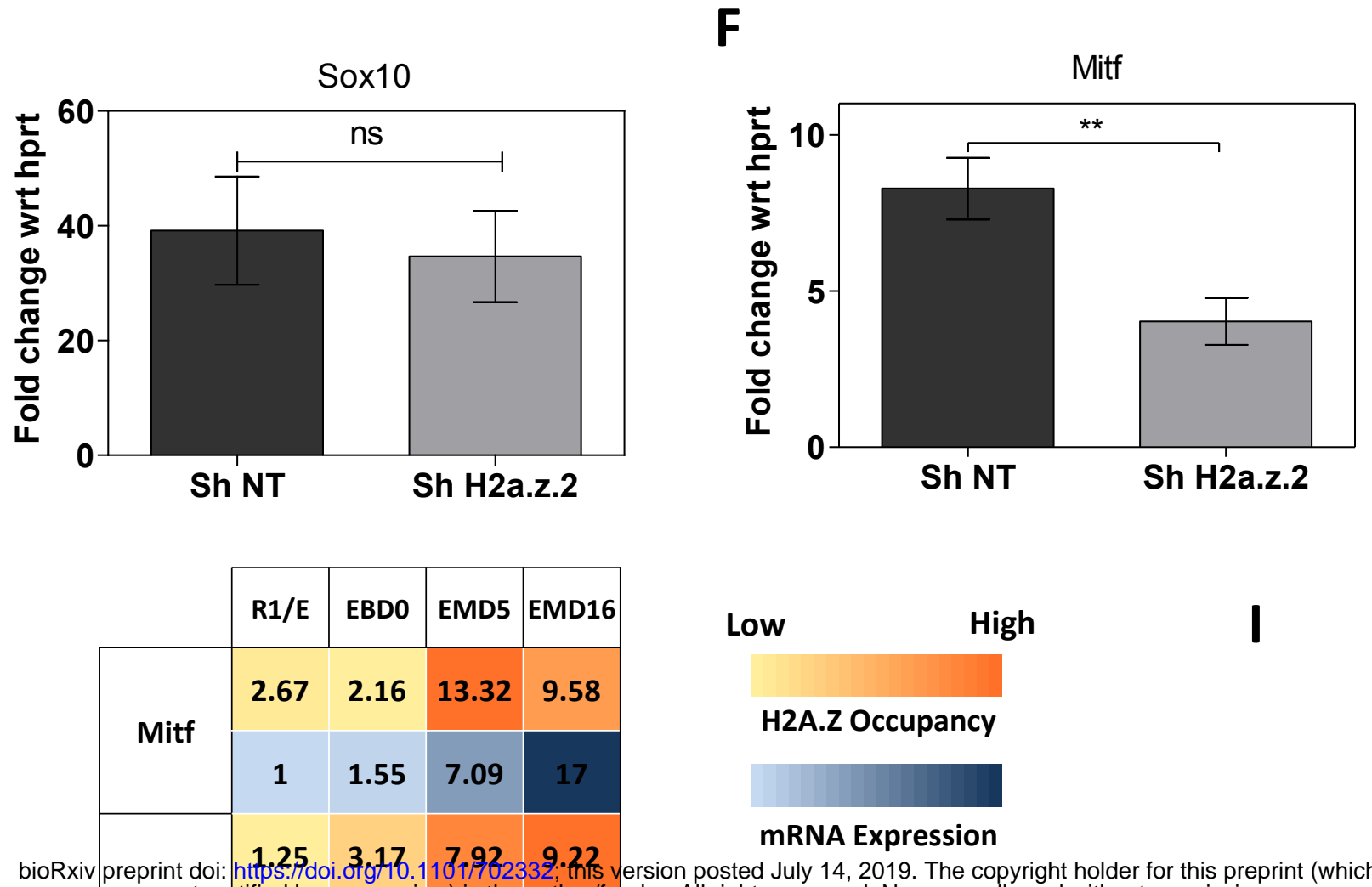

G

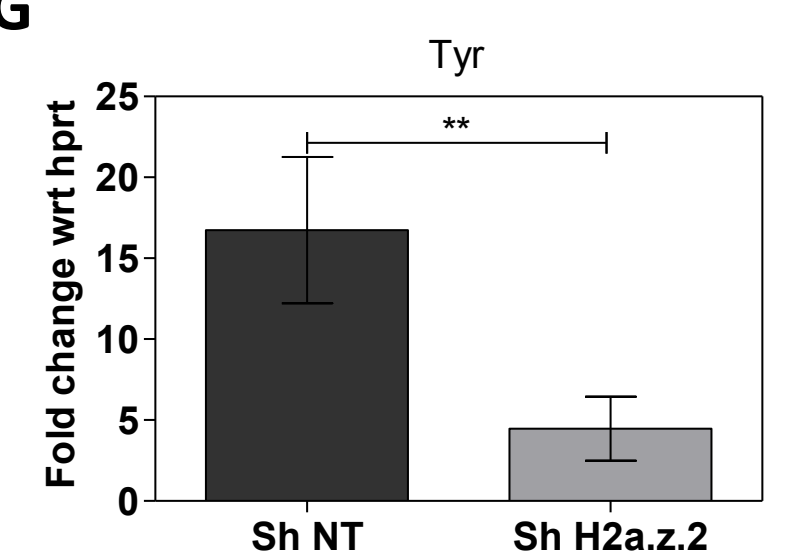

\begin{tabular}{ll} 
Sh NT $\quad$ Sh H2a.z.2 \\
\hline
\end{tabular}

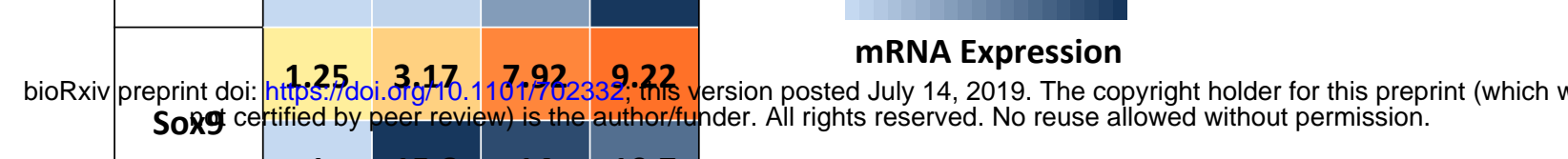

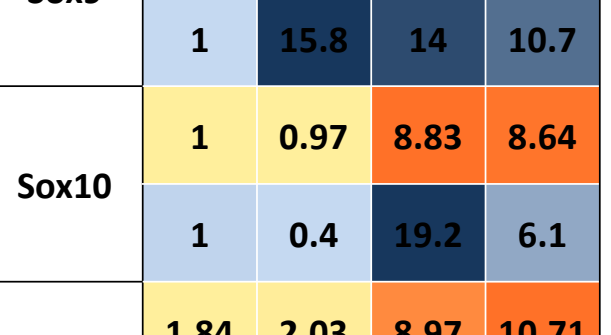

\begin{tabular}{l|l|l|l|l}
1.84 & 2.03 & 8.97 & 10.71
\end{tabular}

\begin{tabular}{l|l|l|l|l|} 
C-kit & 1 & 2.62 & 6.16 & $\mathbf{0 . 9 2}$ \\
\hline
\end{tabular}

$J$

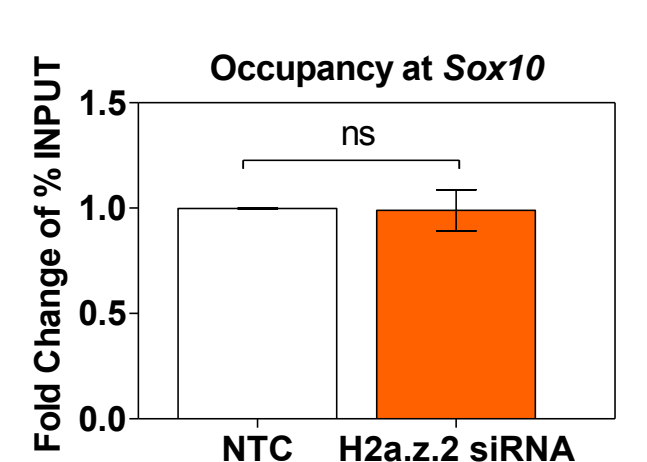

$\mathbf{K}$
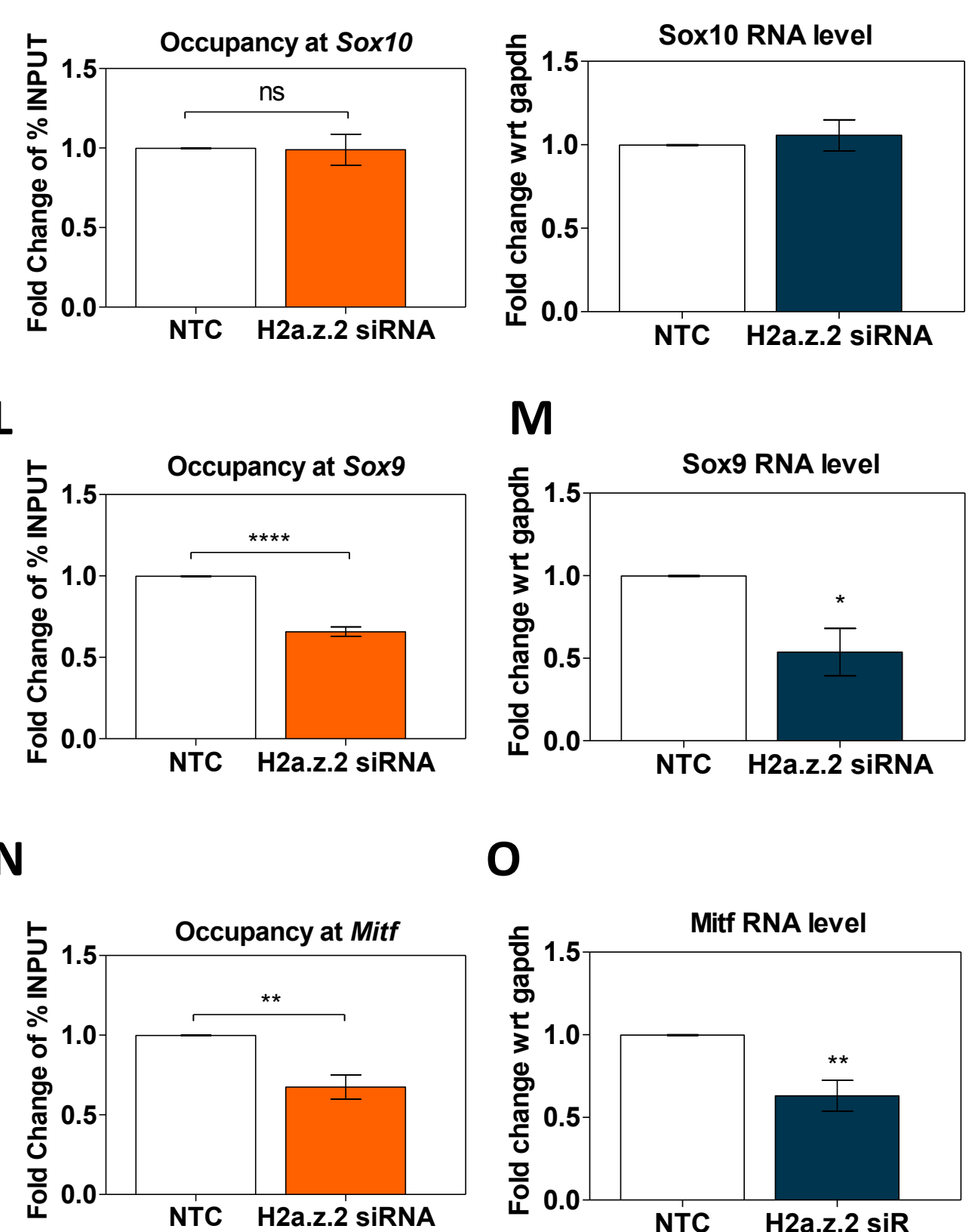

0

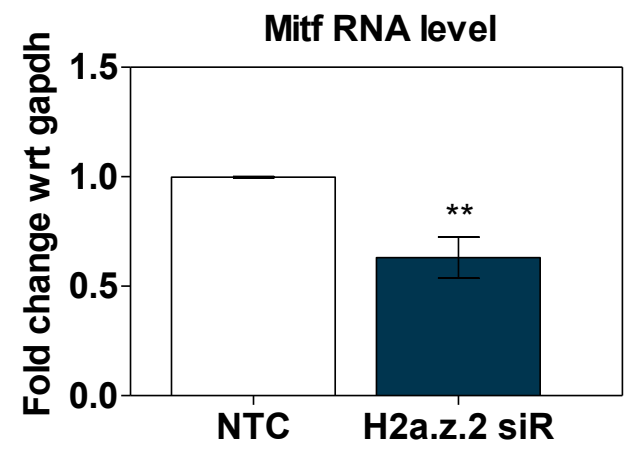

$\mathbf{P}$

Q

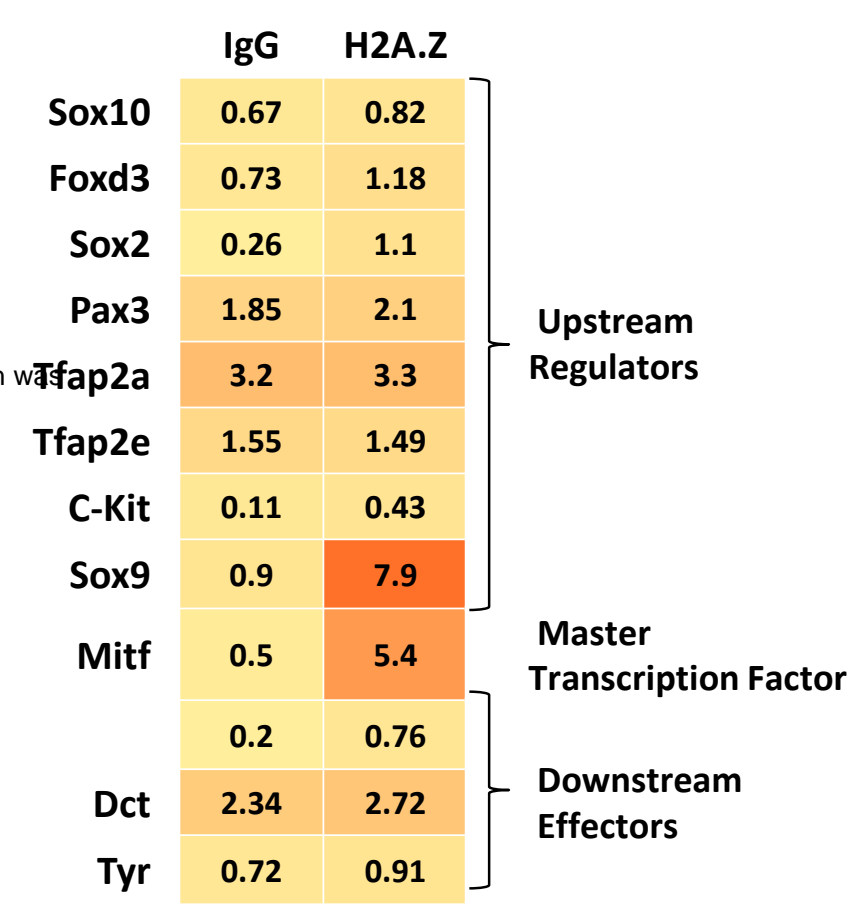

mH2a.z.2

Tubulin

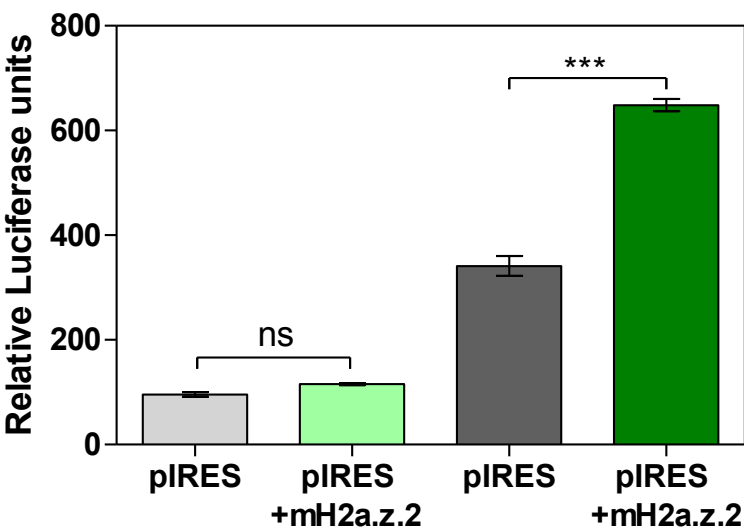

$\alpha-M S$

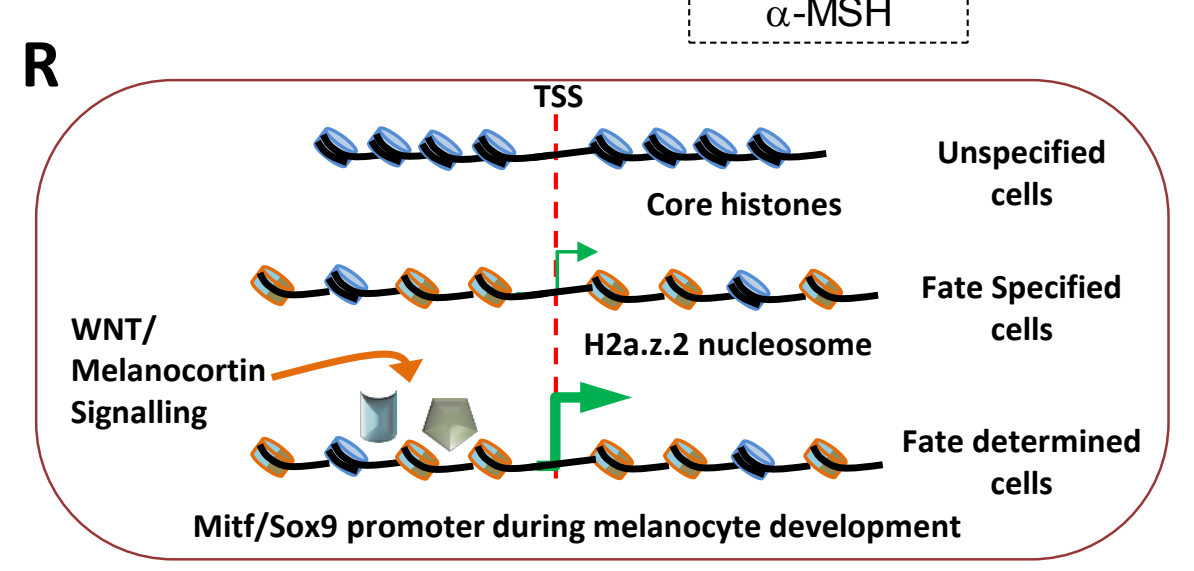


